OHSTPY-HEP-T-09-003

SU-ITP-10-05

NSF-KITP-10-067

\title{
Moduli stabilization and SUSY breaking in heterotic orbifold string models
}

\author{
Ben Dundee $1^{1} a^{a}$, Stuart Raby $2^{2 a \dagger}$, and Alexander Westphal $3^{3 b \dagger}$ \\ a Department of Physics, The Ohio State University, \\ 191 W. Woodruff Ave, Columbus, OH 43210, USA \\ ${ }^{b}$ Department of Physics, Stanford University \\ 382 Via Pueblo Mall, Stanford, CA 94305, USA \\ ${ }^{\dagger}$ Kavli Institute for Theoretical Physics, \\ Santa Barbara, CA 93106, USA
}

\begin{abstract}
In this paper we discuss the issues of supersymmetry breaking and moduli stabilization within the context of $E_{8} \otimes E_{8}$ heterotic orbifold constructions and, in particular, we focus on the class of "minilandscape" models. In the supersymmetric limit, these models admit an effective low energy field theory with a spectrum of states and dimensionless gauge and Yukawa couplings very much like that of the MSSM. These theories contain a non-Abelian hidden gauge sector which generates a non-perturbative superpotential leading to supersymmetry breaking and moduli stabilization. We demonstrate this effect in a simple model which contains many of the features of the more general construction. In addition, we argue that once supersymmetry is broken in a restricted sector of the theory, then all moduli are stabilized by supergravity effects. Finally, we obtain the low energy superparticle spectrum resulting from this simple model.
\end{abstract}

\footnotetext{
${ }^{1}$ Email: dundee@mps . ohio-state.edu

${ }^{2}$ Email: raby@pacific.mps.ohio-state.edu

${ }^{3}$ Email: awestpha@stanford.edu
} 


\section{Introduction}

String theory, as a candidate theory of all fundamental interactions including gravity, is obliged to contain patterns consistent with observation. This includes the standard model gauge group and particle content, as well as an extremely small cosmological constant. If one also assumes that nature contains a low energy supersymmetry, one would want to find patterns which are qualitatively close to the MSSM. An even more ambitious goal would be to find a theory consistent with the standard model spectrum of masses and a prediction for sparticle masses which can be tested at the LHC. Some progress has been made to this end starting from different directions [1, 2, 3, 4, 5, 6, 17, 8, 9, 10, 11, 12, 13, 14, 15, 16, 17, 18, 19, i.e. free fermionic, orbifold or smooth Calabi-Yau constructions of the heterotic string, intersecting Dbrane constructions in type II string, and $\mathrm{M}$ or F theory constructions. Much of this progress has benefited from the requirement of an intermediate grand unified gauge symmetry which naturally delivers the standard model particle spectrum.

In this paper we focus on the "mini-landscape" of heterotic orbifold constructions [4, 5, 6, 9, 10, which give several models which pass a significant number of phenomenological hurdles 1 These models have been analyzed in the supersymmetric limit. They contain an MSSM spectrum with three families of quarks and leptons, one or more pairs of Higgs doublets and an exact $\mathrm{R}$ parity. In the orbifold limit, they also contain a small number of vector-like exotics and extra $U(1)$ gauge interactions felt by standard model particles. These theories also contain a large number of standard model singlet fields, some of which are moduli, i.e. blow up modes of the orbifold fixed points. The superpotential for these orbifold theories can be calculated order by order in powers of products of superfields. This is a laborious task which is simplified by assuming that any term allowed by string selection rules appears with an order one coefficient in the superpotential. With this caveat it was shown that all vector-like exotics and additional $U(1)$ gauge bosons acquire mass at scales of order the string scale at supersymmetric minima satisfying $F_{I}=D_{a}=0$ for all chiral fields labeled by the index $I$ and all gauge groups labeled by the index $a$. In addition, the value of the gauge couplings at the string scale and the effective Yukawa couplings are determined by the presumed values of the vacuum expectation values [VEVs] for mod-

\footnotetext{
${ }^{1}$ For reviews, see [20, 21].
} 
uli including the dilaton, $S$, the bulk volume and complex structure moduli, $T_{i}, i=1,2,3$ and $U$ and the SM singlet fields containing the blow-up moduli [22, 23]. Finally the theories also contain a hidden sector non-Abelian gauge group with QCD-like chiral matter. The problem which has yet to be addressed is the mechanism of moduli stabilization and supersymmetry breaking in the "mini-landscape" models.2

In this paper we focus on the problem of moduli stabilization and SUSY breaking in the context of heterotic orbifold models. In Section 2 we summarize the general structure of the Kähler and superpotential in heterotic orbifold models. The models have a perturbative superpotential satisfying modular invariance constraints, an anomalous $U(1)_{A}$ gauge symmetry with a dynamically generated Fayet-Illiopoulos $D$-term and a hidden QCD-like non-Abelian gauge sector generating a non-perturbative superpotential. In Section 3 we consider a simple model with a dilaton, $S$, one volume modulus, $T$, and three standard model singlets. The model has only one gaugino condensate, as is the case for the "benchmark models" of the "mini-landscape" [9]. We obtain a 'hybrid KKLT' kind of superpotential that behaves like a single-condensate for the dilaton $S$, but as a racetrack for the $T$ and, by extension, also for the $U$ moduli; and an additional matter $F$ term, driven by the cancelation of an anomalous $U(1)_{A} D$-term, is the seed for successful up-lifting. Previous analyses in the literature have also used an anomalous $U(1)_{A} D$-term in coordination with other perturbative or nonperturbative terms in the superpotential to accomplish SUSY breaking and up-lifting [30, 31, 32, 33, 34, 35, 36, 37, 38, 39, 40]. We save a brief comparison of our work with some of these former analyses for Section 3 . In Section 4 we discuss the other moduli and their stabilization. We conclude that a single gaugino condensate is sufficient to break supersymmetry, stabilize all the moduli and generate a de Sitter vacuum. Finally in Section 5 we evaluate the SUSY particle spectrum relevant for the LHC. The main results from this analysis are listed in Tables 7, 8,

\footnotetext{
${ }^{2}$ For a preliminary analysis, see 24. Also moduli stabilization and supersymmetry breaking in Type II string models and F theory constructions have been considered in 25, 26, 27, 28, 29].
} 


\section{General structure}

In this section we consider the supergravity limit of heterotic orbifold models. However, we focus on the "mini-landscape" models for definiteness. We discuss the general structure of the Kähler potential, $\mathcal{K}$, the superpotential, $\mathcal{W}$, and gauge kinetic function, $f_{a}$ for generic heterotic orbifold models. The "mini-landscape" models are defined in terms of a $\mathbb{Z}_{6}$-II orbifold of the six internal dimensions of the ten dimensional heterotic string. The orbifold is described by a three dimensional "twist" vector $v$, which acts on the compact directions. We define the compact directions in terms of complex coordinates:

$$
\begin{aligned}
Z_{1} & \equiv X_{4}+i X_{5}, \\
Z_{2} & \equiv X_{6}+i X_{7}, \\
Z_{3} & \equiv X_{8}+i X_{9} .
\end{aligned}
$$

The twist is defined by the action $Z_{i} \rightarrow e^{2 \pi i v_{i}} Z_{i}$ for $i=1,2,3$, and for $\mathbb{Z}_{6}$-II we have $v=\frac{1}{6}(1,2,-3)$ or a $\left(60^{\circ}, 120^{\circ}, 180^{\circ}\right)$ rotation about the first, second and third torus, respectively. This defines the first twisted sector. The second and fourth twisted sectors are defined by twist vectors $2 v$ and $4 v$, respectively. Note, the third torus is unaffected by this twist. In addition, for the third twisted sector, generated by the twist vector $3 v$, the second torus is unaffected. Finally the fifth twisted sector, given by $5 v$ contains the $C P$ conjugate states from the first twisted sector. Twisted sectors with unrotated tori contain $N=2$ supersymmetric spectra. This has consequences for the non-perturbative superpotential discussed in Section 2.3. Finally, these models have three bulk volume moduli, $T_{i}, i=1,2,3$ and one bulk complex structure modulus, $U$, for the third torus.

\subsection{Anomalous $U(1)_{A}$ and Fayet-Illiopoulos $D$-term}

The orbifold limit of the heterotic string has one anomalous $U(1)_{A}$ symmetry. The dilaton superfield $S$, in fact, transforms non-trivially under this symmetry. Let $V_{A}, V_{a}$ be the gauge superfields with gauge covariant field strengths, $W_{A}^{\alpha}, W_{a}^{\alpha}$, of gauge groups, $U(1)_{A}, \mathcal{G}_{a}$, respectively. The Lagrangian in the

global limit is given in terms of a Kähler potential [41, 42, 43, 44, 45]

$$
\mathcal{K}=-\log \left(S+\bar{S}-\delta_{G S} V_{A}\right)+\sum_{a}\left(\bar{Q}_{a} e^{V_{a}+2 q_{a} V_{A}} Q_{a}+\overline{\tilde{Q}}_{a} e^{-V_{a}+2 \tilde{q}_{a} V_{A}} \tilde{Q}_{a}\right)
$$


and a gauge kinetic superpotential

$$
\mathcal{W}=\frac{1}{2}\left[\frac{S}{4}\left(\sum_{a} k_{a} \operatorname{Tr} W_{a}^{\alpha} W_{\alpha a}+k_{A} \operatorname{Tr} W_{A}^{\alpha} W_{\alpha A}\right)+\text { h.c. }\right] .
$$

Note $q_{a}, \tilde{q}_{a}$ are the $U(1)_{A}$ charges of the 'quark', $Q_{a}$, and 'anti-quark', $\tilde{Q}_{a}$, supermultiplets transforming under $\mathcal{G}_{a}$.

Under a $U(1)_{A}$ super-gauge transformation with parameter $\Lambda$, one has

$$
\begin{aligned}
\delta_{A} V_{A} & =-i(\Lambda-\bar{\Lambda}) / 2, \\
\delta_{A} S & =-i \frac{\delta_{G S}}{2} \Lambda
\end{aligned}
$$

and

$$
\delta_{A} \Phi=i q_{\Phi} \Lambda \Phi
$$

for any charged multiplet $\Phi$. The combination

$$
S+\bar{S}-\delta_{G S} V_{A}
$$

is $U(1)_{A}$ invariant. $\delta_{G S}$ is the Green-Schwarz coefficient given by

$$
\delta_{G S}=4 \frac{\operatorname{Tr} Q_{A}}{192 \pi^{2}}=\frac{\left(q_{a}+\tilde{q}_{a}\right) N_{f_{a}}}{4 \pi^{2}}
$$

where the middle term is for the $U(1)_{A}$-gravity anomaly and the last term is for the $U(1)_{A} \times\left(\mathcal{G}_{a}\right)^{2}$ mixed anomaly.

The existence of an anomalous $U(1)_{A}$ has several interesting consequences. Due to the form of the Kähler potential (Eqn. (2)) we obtain a FayetIlliopoulos $D$-term given by

$$
\xi_{A}=\frac{\delta_{G S}}{2(S+\bar{S})}=-\frac{1}{2} \delta_{G S} \partial_{S} \mathcal{K}
$$

with the $D$-term contribution to the scalar potential given by

$$
V_{D}=\frac{1}{S+\bar{S}}\left(\sum_{a} X_{a}^{A} \partial_{a} \mathcal{K} \phi^{a}+\xi_{A}\right)^{2}
$$

where $X_{a}^{A}$ are Killing vectors for $U(1)_{A}$. In addition, clearly the perturbative part of the superpotential must be $U(1)_{A}$ invariant. But moreover, it constrains the non-perturbative superpotential as well. In particular, if the dilaton appears in the exponent, the product $e^{q_{\Phi} S} \Phi^{\delta_{G S} / 2}$ is, and must also be, $U(1)_{A}$ invariant. 


\subsection{Target space modular invariance}

In this section, we wish to present the modular dependence of the gauge kinetic function, the Kähler potential, and of the superpotential in as general a form as possible. Most studies in the past have worked with a universal $T$ modulus, and neglected the effects of the $U$ moduli altogether. Such a treatment is warranted, for example, in the $\mathbb{Z}_{3}$ orbifolds where there are no $U$ moduli. If we want to work in the limit of a stringy orbifold GUT [46] which requires one of the $T$ moduli to be much larger than the others, or in the $\mathbb{Z}_{6}$-II orbifolds, however, it is impossible to treat all of the $T$ and $U$ moduli on the same footing.

Consider the $S L(2, \mathbb{Z})$ modular transformations of $T$ and $U$ given by [47, 48, 49, 50, 51, 52, 53, 54, 55, 56, 57, 58,3

$$
T \rightarrow \frac{a T-i b}{i c T+d}, a d-b c=1, a, b, c, d \in \mathbb{Z},
$$

and

$$
\log (T+\bar{T}) \rightarrow \log \left(\frac{T+\bar{T}}{(i c T+d)(-i c \bar{T}+d)}\right) .
$$

The Kähler potential for moduli to zeroth order is given by:

$$
\begin{aligned}
\mathcal{K} & =-\sum_{i=1}^{h_{(1,1)}} \log \left(T^{i}+\bar{T}^{i}\right)-\sum_{j=1}^{h_{(2,1)}} \log \left(U^{j}+\bar{U}^{j}\right) \\
& =-\sum_{i=1}^{3} \log \left(T^{i}+\bar{T}^{i}\right)-\log (U+\bar{U})
\end{aligned}
$$

where the last term applies to the "mini-landscape" models, since in this case $h_{(1,1)}=3, h_{(2,1)}=1$. Under the modular group, the Kähler potential transforms as

$$
\mathcal{K} \rightarrow \mathcal{K}+\sum_{i=1}^{h_{(1,1)}} \log \left|i c_{i} T^{i}+d_{i}\right|^{2}+\sum_{j=1}^{h_{(2,1)}} \log \left|i c_{j} U^{j}+d_{j}\right|^{2}
$$

The scalar potential $V$ is necessarily modular invariant. We have

$$
V=e^{\mathcal{G}}\left(\mathcal{G}_{I} \mathcal{G}^{I \bar{J}} \mathcal{G}_{\bar{J}}-3\right)
$$

\footnotetext{
${ }^{3}$ For an excellent review with many references, see [59].
} 
where $\mathcal{G}=\mathcal{K}+\log |\mathcal{W}|^{2}$. Hence for the scalar potential to be invariant under the modular transformations, the superpotential must also transform as follows:

$$
\begin{gathered}
\mathcal{W} \rightarrow \prod_{i=1}^{h_{(1,1)}} \prod_{j=1}^{h_{(2,1)}}\left(i c_{i} T^{i}+d_{i}\right)^{-1}\left(i c_{j} U^{j}+d_{j}\right)^{-1} \mathcal{W} \\
\overline{\mathcal{W}} \rightarrow \prod_{i=1}^{h_{(1,1)}} \prod_{j=1}^{h_{(2,1)}}\left(-i c_{i} \bar{T}^{i}+d_{i}\right)^{-1}\left(-i c_{j} \bar{U}^{j}+d_{j}\right)^{-1} \overline{\mathcal{W}} .
\end{gathered}
$$

This can be guaranteed by appropriate powers of the Dedekind $\eta$ function multiplying terms in the superpotential 4 This is due to the fact that under a modular transformation, we have

$$
\eta(T) \rightarrow(i c T+d)^{1 / 2} \eta(T)
$$

up to a phase, where

$$
\eta(T)=\exp (-\pi T / 12) \prod_{n=1}^{\infty}\left(1-e^{-2 \pi n T}\right) .
$$

The transformation of both the matter fields and the superpotential under the modular group fixes the modular dependence of the interactions. A field in the superpotential transforms as

$$
\Phi_{I} \rightarrow \Phi_{I} \prod_{i=1}^{h_{(1,1)}} \prod_{j=1}^{h_{(2,1)}}\left(i c_{i} T^{i}+d_{i}\right)^{-n_{I}^{i}}\left(i c_{j} U^{j}+d_{j}\right)^{-\ell_{I}^{j}}
$$

The modular weights $n_{I}^{i}$ and $\ell_{I}^{j}$ [60, 61] depend on the localization of the matter fields on the orbifold. For states $I$ in the $i$ th untwisted sector, i.e. those states with internal momentum in the $i$ th torus, we have $n_{I}^{i}=\ell_{I}^{i}=1$, otherwise the weights are 0 . For twisted sector states, we first define $\vec{\eta}(k)$, which is related to the twisted sector $k(=1, \ldots, N-1)$ and the orbifold twist vector $v$ by

$$
\eta_{i}(k) \equiv k v_{i} \quad \bmod 1 .
$$

\footnotetext{
${ }^{4}$ These terms arise as a consequence of world-sheet instantons in a string calculation. In fact, world sheet instantons typically result in more general modular functions [52, 53 , 54, 55, 56, 57, 58,
} 
Further, we require

$$
\sum_{i} \eta_{i}(k) \equiv 1 .
$$

Then the modular weight of a state in the $k$ th twisted sector is given by

$$
\begin{aligned}
& n_{I}^{i} \equiv\left(1-\eta^{i}(k)\right)+N^{i}-\bar{N}^{i} \text { for } \eta_{i}(k) \neq 0 \\
& n_{I}^{i} \equiv \quad N^{i}-\bar{N}^{i} \quad \text { for } \eta_{i}(k)=0 \text {. }
\end{aligned}
$$

The $N^{i}\left(\bar{N}^{i}\right)$ are integer oscillator numbers for left-moving oscillators $\tilde{\alpha}^{i}\left(\overline{\tilde{\alpha}}^{\bar{\alpha}}\right)$, respectively. Similarly,

$$
\begin{array}{lcc}
\ell_{I}^{i} \equiv & \left(1-\eta^{i}(k)\right)-N^{i}+\bar{N}^{i} & \text { for } \eta_{i}(k) \neq 0 \\
\ell_{I}^{i} \equiv & -N^{i}+\bar{N}^{i} & \text { for } \eta_{i}(k)=0 .
\end{array}
$$

In general, one can compute the superpotential to arbitrary order in powers of superfields by a straightforward application of the string selection rules $[62,63,64,65]$. One assumes that any term not forbidden by the string selection rules appears with order one coefficient. In practice, even this becomes intractable quickly, and we must cut off the procedure at some low, finite order. More detailed calculations of individual terms give coefficients dependent on volume moduli due to string world sheet instantons. In general the moduli dependence can be obtained using the constraint of target space modular invariance. Consider a superpotential term for the "mini-landscape" models, with three $T$ moduli and one $U$ modulus, of the form:

$$
\mathcal{W}_{3}=w_{I J K} \Phi_{I} \Phi_{J} \Phi_{K} .
$$

We assume that the fields $\Phi_{I, J, K}$ transform with modular weights $n_{I, J, K}^{i}$ and $\ell_{I, J, K}^{3}$ under $T_{i}, i=1,2,3$ and $U$, respectively. Using the (net) transformation property of the superpotential, and the transformation property of $\eta(T)$ under the modular group, we have (for non-universal moduli):

$$
w_{I J K} \sim h_{I J K} \prod_{i=1}^{3} \eta\left(T_{i}\right)^{\gamma_{T_{i}}} \eta(U)^{\gamma_{U}}
$$

where $\gamma_{T_{i}}=-2\left(1-n_{I}^{i}-n_{J}^{i}-n_{K}^{i}\right), \gamma_{U}=-2\left(1-\ell_{I}^{3}-\ell_{J}^{3}-\ell_{K}^{3}\right)$.5 This is easily generalized for higher order interaction terms in the superpotential. We see

\footnotetext{
${ }^{5}$ Note, the constants $\gamma_{T_{i}}, \gamma_{U}$ can quite generally have either sign, depending upon the modular weights of the fields at the particular vertex.
} 
that the modular dependence of the superpotential is rarely symmetric under interchange of the $T_{i}$ or $U_{i}$. Note, when minimizing the scalar potential we shall use the approximation $\eta(T)^{\gamma_{T}} \approx e^{-b T}$ with $b=\pi \gamma_{T} / 12$. (Recall, at large $T$, we have $\log (\eta(T)) \approx-\pi T / 12$.) This approximation misses the physics near the self-dual point in the potential, nevertheless, it is typically a good approximation.

As a final note, Wilson lines break the $S L(2, \mathbb{Z})$ modular group down to a subgroup [66] (see Appendix A). This has the effect of an additional differentiation of the moduli as they appear in the superpotential. In particular, factors of $\eta\left(T_{i}\right)$ are replaced by factors of $\eta\left(N T_{i}\right)$ or $\eta\left(T_{i} / N\right)$ for Wilson lines in $\mathbb{Z}_{N}$. In summary, the different modular dependence of twisted sector fields and the presence of Wilson lines leads quite generally to anisotropic orbifolds 67 .

\subsection{Gauge kinetic function and sigma model anomaly}

To one loop, the string-derived gauge kinetic function is given by [68, 70,69 , 71, 61, 72

$$
\begin{aligned}
f_{a}(S, T)= & k_{a} S+\frac{1}{8 \pi^{2}} \sum_{i=1}^{h_{(1,1)}}\left(\alpha_{a}^{i}-k_{a} \delta_{\sigma}^{i}\right) \log \left(\eta\left(T^{i}\right)\right)^{2} \\
& +\frac{1}{8 \pi^{2}} \sum_{j=1}^{h_{(2,1)}}\left(\alpha_{a}^{j}-k_{a} \delta_{\sigma}^{j}\right) \log \left(\eta\left(U^{j}\right)\right)^{2}
\end{aligned}
$$

where $k_{a}$ is the Kač-Moody level of the group, which we will normally take to be 1 . The constants $\alpha_{a}^{i}$ are model dependent, and are defined as

$$
\alpha_{a}^{i} \equiv \ell(\operatorname{adj})-\sum_{\operatorname{rep}_{I}} \ell_{a}\left(\operatorname{rep}_{I}\right)\left(1+2 n_{I}^{i}\right)
$$

$\ell(\operatorname{adj})$ and $\ell_{a}\left(\operatorname{rep}_{I}\right)$ are the Dynkin indices of the adjoint representation and of the matter representation $I$ of the group $\mathcal{G}_{a}$, respectively [73] and $n_{I}^{i}$ are modular weights 6 The $\delta_{\sigma}^{i}$ terms are necessary to cancel an anomaly in the underlying $\sigma$-model, which induces a transformation in the dilaton field under

\footnotetext{
${ }^{6}$ If $T_{a}^{r}$ are the generators of the group $G_{a}$ in the representation $r$, then we have $\operatorname{Tr}\left(T_{a}^{r} T_{b}^{r}\right)=\ell_{a}\left(\operatorname{rep}_{r}\right) \delta_{a b}$.
} 
the modular group:

$$
S \rightarrow S+\frac{1}{8 \pi^{2}} \sum_{i=1}^{h_{(1,1)}} \delta_{\sigma}^{i} \log \left(i c_{i} T_{i}+d_{i}\right)+\frac{1}{8 \pi^{2}} \sum_{j=1}^{h_{(2,1)}} \delta_{\sigma}^{i} \log \left(i c_{j} U^{j}+d_{j}\right) .
$$

It is important to note that the factor

$$
\left(\alpha_{a}^{i}-k_{a} \delta_{\sigma}^{i}\right) \equiv \frac{b_{a}^{(N=2)}(i)}{|D| /\left|D_{i}\right|}
$$

where $b_{a}^{(N=2)}(i)$ is the beta function coefficient for the $i$ th torus. It is non-zero if and only if the $k$-th twisted sector has an effective $N=2$ supersymmetry. Moreover this occurs only when, in the $k$-th twisted sector, the $i$ th torus is not rotated. The factors $|D|,\left|D_{i}\right|$ are the degree of the twist group $D$ and the little group $D_{i}$, which does not rotate the $i$ th torus. For example, for the "mini-landscape" models with $D=\mathbb{Z}_{6}$-II we have $|D|=6$ and $\left|D_{2}\right|=2,\left|D_{3}\right|=3$ since the little group keeping the second (third) torus fixed is $\mathbb{Z}_{2}\left(\mathbb{Z}_{3}\right)$. The first torus is rotated in all twisted sectors. Hence, the gauge kinetic function for the "mini-landscape" models is only a function of $T_{2}$ and $T_{3}$.

Taking into account the sigma model anomalies, the heterotic string Kähler potential has the following form, where we have included the loop corrections to the dilaton [68, 70]

$$
\begin{aligned}
\mathcal{K}= & -\log \left(S+\bar{S}+\frac{1}{8 \pi^{2}} \sum_{i=1}^{h_{(1,1)}} \delta_{\sigma}^{i} \log \left(T^{i}+\bar{T}^{i}\right)+\frac{1}{8 \pi^{2}} \sum_{j=1}^{h_{(2,1)}} \delta_{\sigma}^{j} \log \left(U^{j}+\bar{U}^{j}\right)\right) \\
& -\sum_{i=1}^{h_{(1,1)}} \log \left(T^{i}+\bar{T}^{i}\right)-\sum_{j=1}^{h_{(2,1)}} \log \left(U^{j}+\bar{U}^{j}\right) .
\end{aligned}
$$

The first line of Eqn. (27) is modular invariant by itself, and one can redefine the dilaton, $Y$, such that

$$
Y \equiv S+\bar{S}+\frac{1}{8 \pi^{2}} \sum_{i=1}^{h_{(1,1)}} \delta_{\sigma}^{i} \log \left(T^{i}+\bar{T}^{i}\right)+\frac{1}{8 \pi^{2}} \sum_{j=1}^{h_{(1,2)}} \delta_{\sigma}^{j} \log \left(U^{j}+\bar{U}^{j}\right)
$$

where $Y$ is invariant under the modular transformations. 
Table 1: Charge assignments for the fields in a generic hidden sector. Flavor indices are suppressed.

\begin{tabular}{c|cccccc} 
& $\phi$ & $\chi$ & $Q_{1}$ & $Q_{2}$ & $\tilde{Q}_{1}$ & $\tilde{Q}_{2}$ \\
\hline $\mathrm{U}(1)_{A}$ & -1 & $q_{\chi}$ & $q_{1}$ & $q_{2}$ & $\tilde{q}_{1}$ & $\tilde{q}_{2}$ \\
$\mathrm{SU}\left(N_{1}\right)$ & 1 & 1 & $\square$ & 1 & $\square$ & 1 \\
$\mathrm{SU}\left(N_{2}\right)$ & 1 & 1 & 1 & $\square$ & 1 & $\square$
\end{tabular}

\subsection{Non-perturbative superpotential}

In all "mini-landscape" models 24, and most orbifold heterotic string constructions, there exists a hidden sector with non-Abelian gauge interactions and vector-like matter carrying hidden sector charge. In the "benchmark" models [9] the hidden sector gauge group is $S U(4)$ with chiral matter in the $4+\overline{4}$ representation.

In this section let us consider a generic hidden sector with gauge group $S U\left(N_{1}\right) \otimes S U\left(N_{2}\right) \otimes U(1)_{A}$, where ' $A$ ' stands for anomalous. There are $N_{f_{1}}$ and $N_{f_{2}}$ flavors of quarks $Q_{1}$ and $Q_{2}$ in the fundamental representation (along with anti-quarks $\tilde{Q}_{1}$ and $\tilde{Q}_{2}$, in the anti-fundamental representations), as well as two singlet fields, called $\phi$ and $\chi$. The charge assignments are listed in Table 1. We assume the existence of two moduli, $S$ and $T$, which enter the non-perturbative superpotential through the gauge kinetic function, namely $f=f(S, T)$. The model also allows for $T$ dependence in the Yukawa sector.

Non-perturbative effects generate a potential for the $S$ and $T$ moduli. Gaugino condensation will generate a scale $\Lambda_{\mathrm{SQCD}}$, which is determined purely by the symmetries of the low energy theory:

$$
\Lambda_{a}(S, T)=e^{-\frac{8 \pi^{2}}{\beta_{a}} f_{a}(S, T)},
$$

where $\beta_{a}=3 N_{a}-N_{f_{a}}$ is the one loop beta function coefficient of the theory. At tree level $f_{a}(S, T)=S$, however, we include the possibility of threshold corrections which introduce a dependence on the $T$ modulus [68, 70]. We also find that $U(1)_{A}$ and modular invariance together dictate a very specific form for the non-perturbative superpotential.

In the "mini-landscape" analysis the effective mass terms for the vectorlike exotics were evaluated. They were given as a polynomial in products of chiral MSSM singlet fields [chiral moduli]. It was shown that all vector-like exotics obtain mass 7 when the chiral moduli obtain VEVs at supersymmetric

\footnotetext{
${ }^{7}$ In fact, one of the $S U(4)$ quark- anti-quark pairs remained massless in the two "bench-
} 
points in moduli space. In our example let us, for simplicity, take couplings between the quarks and the field $\phi$ to be diagonal in flavor space. Mass terms of the form

$$
\mathbb{M}_{1}(\phi, T) Q_{1} \tilde{Q}_{1}+\mathbb{M}_{2}(\phi, T) Q_{2} \tilde{Q}_{2}
$$

are dynamically generated when $\phi$ receives a non-zero $\mathrm{VEV}$, which we will discuss below. A key assumption is that those mass terms are larger than the scale of gaugino condensation, so that the quarks and anti-quarks may be consistently integrated out. If this can be accomplished, then one can work in the pure gauge limit [74].8

Before we integrate out the meson fields, the non-perturbative superpotential (plus quark masses) for $N_{f_{a}}<N_{a}$ is of the form [75]

$$
\mathcal{W}_{\mathrm{NP}}=\sum_{a=1,2}\left[\mathbb{M}_{a}(\phi, T) Q_{a} \tilde{Q}_{a}+\left(N_{a}-N_{f_{a}}\right)\left(\frac{\Lambda_{a}^{3 N_{a}-N_{f_{a}}}}{\operatorname{det} Q_{a} \tilde{Q}_{a}}\right)^{\frac{1}{N_{a}-N_{f_{a}}}}\right]
$$

with $\mathbb{M}_{a}(\phi, T)=c_{a} e^{-b_{a} T} \phi^{q_{a}+\tilde{q}_{a}}$ where $c_{a}$ is a constant. Note, given the charges for the fields in Table 1 and using Eqns. (41), (7) and (29), one sees that $\mathcal{W}_{\mathrm{NP}}$ is $U(1)_{A}$ invariant. The Kähler potential for the hidden sector is assumed to be of the form

$$
\begin{gathered}
\mathcal{K}=-\log (S+\bar{S})-3 \log (T+\bar{T})+\alpha_{\phi} \bar{\phi} e^{-2 V_{A} \phi}+\alpha_{\chi} \bar{\chi} e^{2 q_{\chi} V_{A}} \chi \\
+\sum_{a=1,2} \alpha_{a}\left(\bar{Q}_{a} e^{V_{a}+2 q_{a} V_{A}} Q_{a}+\overline{\tilde{Q}}_{a} e^{-V_{a}+2 \tilde{q}_{a} V_{A}} \tilde{Q}_{a}\right.
\end{gathered}
$$

The quantities $\alpha_{\phi}, \alpha_{\chi}, \alpha_{i}$ are generally functions of the modulus $T$, where the precise functional dependence is fixed by the modular weights of the fields (see Section 2.2). $V_{i}$ and $V_{A}$ denote the vector superfields associated with the gauge groups $\mathcal{G}_{i}=S U\left(N_{i}\right)$ and $U(1)_{A}$.

The determinant of the quark mass matrix is given by

$$
\operatorname{det} \mathbb{M}_{a}(\phi, T)=\left(c_{a} e^{-b_{a} T} \phi^{q_{a}+\tilde{q}_{a}}\right)^{N_{f_{a}}} .
$$

We have taken the couplings between $\phi$ and the quarks to have exponential dependence on the $T$ modulus, an ansatz which is justified by modular invariance (see Section 2.2). Inserting the meson equations of motion and Eqn.

mark" models.

${ }^{8}$ There is a check on the consistency of this approach: at the end of the day, after calculating the VEVs of the scalars, we can verify that the mass terms for the quarks are indeed of the correct magnitude. 
(33) into Eqn. (31), we have

$$
\mathcal{W}_{\mathrm{NP}}=\sum_{a=1,2}\left[N_{a}\left(c_{a} e^{-b_{a} T} \phi^{q_{a}+\tilde{q}_{a}}\right)^{\frac{N_{f_{a}}}{N_{a}}}\left[\Lambda_{a}(S, T)\right]^{\frac{3 N_{a}-N_{f_{a}}}{N_{a}}}\right] .
$$

Note that the transformation of the superpotential under the modular group in Eqn. (15) also requires that the (non-perturbative) superpotential obey

$$
\mathcal{W}_{\mathrm{NP}} \rightarrow \prod_{i=1}^{h_{(1,1)}} \prod_{j=1}^{h_{(2,1)}}\left(i c_{i} T^{i}+d_{i}\right)^{-1}\left(i c_{j} U^{j}+d_{j}\right)^{-1} \mathcal{W}_{\mathrm{NP}}
$$

Because the non-perturbative lagrangian must be invariant under all of the symmetries of the underlying string theory, it must be that [76, 77, 78, 79, 71, 80]:

$$
\mathcal{W}_{\mathrm{NP}} \equiv A \times e^{-a S} \prod_{i=1}^{h_{(1,1)}} \prod_{j=1}^{h_{(2,1)}}\left(\eta\left(T^{i}\right)\right)^{-2+\frac{3}{4 \pi^{2} \beta} \delta_{\sigma}^{i}}\left(\eta\left(U^{j}\right)\right)^{-2+\frac{3}{4 \pi^{2} \beta} \delta_{\sigma}^{j}}
$$

where $a \equiv \frac{24 \pi^{2}}{\beta}$ and $\beta=3 \ell(\operatorname{adj})-\sum_{I} \ell\left(\operatorname{rep}_{I}\right)$ is the one-loop beta function coefficient, and $A$ is generally a function of the chiral matter fields appearing in $\mathbb{M}$. This, coupled with the one loop gauge kinetic function in Eqn. (24), gives the heterotic generalization of the Racetrack superpotential.

In the following Section 3, we construct a simple model using the qualitative features outlined in this section. This model is novel because it requires only one non-Abelian gauge group to stabilize moduli and give a de Sitter vacuum. We have also constructed two condensate models, however, the literature already contains several examples of the "racetrack" in regards to stabilization of $S$ and $T$ moduli. Moreover in the "mini-landscape" models, whose features we are seeking to reproduce, there are many examples of hidden sectors containing a single non-Abelian gauge group [24], while there are no examples with multiple hidden sectors.

\section{Moduli stabilization and supersymmetry breaking in the bulk}

In this section we construct a simple, generic heterotic orbifold model which captures many of the features discussed in Section 2. In particular, it is 
a single gaugino condensate model with the following fields - dilaton $(S)$, modulus $(T)$ and MSSM singlets $\left(\phi_{1}, \phi_{2}, \chi\right)$. The model has one anomalous $\mathrm{U}(1)_{A}$ with the singlet charges given by $\left(q_{\phi_{1}}=-2, q_{\phi_{2}}=-9, q_{\chi}=20\right)$. The Kähler and superpotential are given by 9

$$
\begin{aligned}
\mathcal{K} & =-\log [S+\bar{S}]-3 \log [T+\bar{T}]+\bar{\phi}_{1} \phi_{1}+\bar{\phi}_{2} \phi_{2}+\bar{\chi} \chi \\
\mathcal{W} & =e^{-b T}\left(w_{0}+\chi\left(\phi_{1}^{10}+\lambda \phi_{1} \phi_{2}^{2}\right)\right)+A \phi_{2}^{p} e^{-a S-b_{2} T} .
\end{aligned}
$$

In addition, there is an anomalous $U(1)_{A} D$-term given by

$$
D_{A}=20 \bar{\chi} \chi-2 \bar{\phi}_{1} \phi_{1}-9 \bar{\phi}_{2} \phi_{2}-\frac{1}{2} \delta_{G S} \partial_{S} \mathcal{K}
$$

with $\delta_{G S}=\frac{(q+\tilde{q}) N_{f}}{4 \pi^{2}}=N_{f} /\left(4 \pi^{2}\right)$.

In the absence of the non-perturbative term (with coefficient $A$ ) the theory has a supersymmetric minimum with $\langle\chi\rangle=\left\langle\phi_{1}\right\rangle=0$ and $\left\langle\phi_{2}\right\rangle \neq 0$ and arbitrary. This property mirrors the situation in the "mini-landscape" models where supersymmetric vacua have been found in the limit that all non-perturbative effects are neglected. We have also added a constant $w_{0}=$ $w_{0}\left(\left\langle\phi_{I}\right\rangle\right)$ which is expected to be generated (in the "mini-landscape" models) at high order in the product of chiral moduli due to the explicit breaking of an accidental $R$ symmetry which exists at lower orders [81] 10 The $T$ dependence in the superpotential is designed to take into account, in a qualitative way, the modular invariance constraints of Section 2.2. We have included only one $T$ modulus, assuming that the others can be stabilized near the self-dual point [76, 82]. Moreover, as argued earlier, the $T_{i}$ and $U$ moduli enter the superpotential in different ways (see Section 2.2). This leads to modular invariant solutions which are typically anisotropic [67, 11

Note, that the structure, $\mathcal{W} \sim w_{0} e^{-b T}+\phi_{2} e^{-a S-b_{2} T}$ gives us the crucial $\operatorname{progres}: 12$ -

\footnotetext{
${ }^{9}$ The coefficient $A$ (Eqn. (37) ) is an implicit function of all other non-vanishing chiral singlet $\mathrm{VEVs}$ which would be necessary to satisfy the modular invariance constraints, i.e. $A=A\left(\left\langle\phi_{I}\right\rangle\right)$. If one re-scales the $U(1)_{A}$ charges, $q_{\phi_{i}}, q_{\chi} \rightarrow q_{\phi_{i}} / r, q_{\chi} / r$, then the $U(1)_{A}$ constraint is satisfied with $r=15 p$ (assuming no additional singlets in $A$ ). Otherwise we may let $r$ and $p$ be independent. This re-scaling does not affect our analysis, since the vacuum value of the $\phi_{i}, \chi$ term in the superpotential vanishes.

${ }^{10}$ The fields entering $w_{0}$ have string scale mass.

${ }^{11}$ Note, we have chosen to keep the form of the Kähler potential for this single $T$ modulus with the factor of 3 , so as to maintain the approximate no-scale behavior.

${ }^{12}$ Note, the constants $b, b_{2}$ can have either sign. For the case with $b, b_{2}>0$ the superpotential for $T$ is racetrack-like. However for $b, b_{2}<0$ the scalar potential for $T$ diverges as $T$ goes to zero or infinity and compactification is guaranteed [76, 82,
} 
i.) a 'hybrid KKLT' kind of superpotential that behaves like a singlecondensate for the dilaton $S$, but as a racetrack for the $T$ and, by extension, also for the $U$ moduli; and

ii.) an additional matter $F_{\phi_{2}}$ term driven by the cancelation of the anomalous $U(1)_{A} D$-term seeds SUSY breaking with successful uplifing.

The constant $b$ is fixed by modular invariance constraints. In general the two terms in the perturbative superpotential would have different $T$ dependence. We have found solutions for this case as well. This is possible since the VEV of the $\chi$ term in the superpotential vanishes. The second term (proportional to $A$ ) represents the non-perturbative contribution of one gaugino condensate. The constants $a=24 \pi^{2} / \beta, b_{2}$ and $p$ depend on the size of the gauge group, the number of flavors and the coefficient of the one-loop beta function for the effective $N=2$ supersymmetry of the torus $T$. For the "mini-landscape" models, this would be either $T_{2}$ or $T_{3}$. Finally, the coefficient of the exponential factor of the dilaton $S$ is taken to be $A \phi_{2}^{p}$. This represents the effective hidden sector quark mass term, which in this case is proportional to a power of the chiral singlet $\phi_{2}$. In a more general case, it would be a polynomial in powers of chiral moduli 13 The exponent $p$ depends in general on the size of the gauge group, the number of flavors and the power that the field $\phi_{2}$ appears in the effective quark mass term.

We have performed a numerical evaluation of the scalar potential with the following input parameters. We take hidden sector gauge group $S U(N)$ with $N=5, N_{f}=3$ and $a=8 \pi^{2} / N 14$ For the other input values we have considered five different possibilities given in Table 2 $\left.2\right|^{15}$ We find that supersymmetry breaking, moduli stabilization and up-lifting is a direct consequence of adding the non-perturbative superpotential term.

\footnotetext{
${ }^{13}$ Holomorphic gauge invariant monomials span the moduli space of supersymmetric vacua. One such monomial is necessary to cancel the Fayet-Illiopoulos $D$-term (see Appendix (B).

${ }^{14}$ We have also found solutions for the case with $N=4, N_{f}=7$ which is closer to the "mini-landscape" benchmark models. Note, when $N_{f}>N$ we may still use the same formalism, since we assume that all the $Q, \tilde{Q}_{\mathrm{s}}$ get mass much above the effective QCD scale.

${ }^{15}$ Note the parameter relation $r=15 p$ in Table 2 is derived using $U(1)_{A}$ invariance and the assumption that no other fields with non-vanishing $U(1)_{A}$ charge enter into the effective mass matrix for hidden sector quarks. We have also allowed for two cases where this relation is not satisfied.
} 
In our analysis we use the scalar potential $V$ given by

$$
V=e^{K}\left(\sum_{i=1}^{5} \sum_{j=1}^{5}\left[F_{\Phi_{i}} \overline{F_{\Phi_{j}}} \mathcal{K}_{i, j}^{-1}-3|W|^{2}\right]\right)+\frac{D_{A}^{2}}{(S+\bar{S})}+\Delta V_{C W}\left[\Phi_{i}, \bar{\Phi}_{i}\right]
$$

where $\Phi_{i, j}=\left\{S, T, \chi, \phi_{1}, \phi_{2}\right\}$ and $F_{\Phi_{i}} \equiv \partial_{\Phi_{i}} \mathcal{W}+\left(\partial_{\Phi_{i}} \mathcal{K}\right) \mathcal{W}$. The first two terms are the tree level supergravity potential. The last term is a one loop correction which affects the vacuum energy and $D$ term contribution.

The one loop Coleman-Weinberg potential is in general given by

$$
\Delta V_{C W}=\frac{1}{32 \pi^{2}} \operatorname{Str}\left(M^{2}\right) \Lambda^{2}+\frac{1}{64 \pi^{2}} \operatorname{Str}\left(M^{4} \log \left[\frac{M^{2}}{\Lambda^{2}}\right]\right)
$$

with the mass matrix $M$ given by $M=M\left(\Phi_{i}\right)$ and $\Lambda$ is the relevant cut-off in the problem. We take $\Lambda=M_{\mathrm{S}} \sim 10^{17} \mathrm{GeV}$.

We have not evaluated the full one loop correction. Instead we use the approximate formula

$$
\Delta V_{C W}\left[\phi_{2}, \overline{\phi_{2}}\right]=\frac{\lambda^{2} F_{2}^{2}\left|\phi_{2}\right|^{2}}{8 \pi^{2}}\left(\log \left[R\left(\lambda\left|\phi_{2}\right|^{2}\right)^{2}\right]+3 / 2\right)+\mathscr{O}\left(\Lambda^{2}\right)
$$

where $F_{2}=\left\langle F_{\phi_{2}}\right\rangle$ is obtained self-consistently and all dimensionful quantities are expressed in Planck units. This one loop expression results from the $\chi, \phi_{1}$ contributions to the Coleman-Weinberg formula. The term quadratic in the cut-off is naturally proportional to the number of chiral multiplets in the theory and could be expected to contribute a small amount to the vacuum energy, of order a few percent times $m_{3 / 2}^{2} M_{p l}^{2}$. We will discuss this contribution later, after finding the minima of the potential. Finally, note that the parameters $\lambda, R$ in Table 2 might both be expected to be significantly greater than one when written in Planck units. This is because the scale of the effective higher dimensional operator with coefficient $\lambda$ in Eqn. 37 is most likely set by some value between $M_{P l}$ and $M_{\text {string }}$ and the cut-off scale for the one loop calculation (which determines the constant $R$ ) is the string scale and not $M_{P l}$.

In all cases we find a meta-stable minimum with all (except for two massless modes) fields massive of $\mathscr{O}(\mathrm{TeV})$ or larger. Supersymmetry is broken at the minimum with values given in Table 3 . Note $\operatorname{Re} S \sim 2.2$ and $\operatorname{Re} T$ ranges between 1.1 and 1.6. The moduli $\chi, \phi_{1}$ are stabilized at their global minima $\phi_{1}=\chi=0$ with $F_{\chi}=F_{\phi_{1}}=0$ in all cases. The modulus $\sigma=\operatorname{Im} S$ 


\begin{tabular}{|c|c|c|c|c|c|c|c|c|}
\hline Case & $b$ & $b_{2}$ & $\lambda$ & $R$ & $p$ & $r$ & $A$ & $w_{0}$ \\
\hline 1 & $\pi / 50$ & $3 \pi / 2$ & 33 & 10 & $2 / 5$ & $15 p$ & 160 & $8 \times 10^{-15}$ \\
\hline 2 & $8 / 125$ & $3 \pi / 2$ & 0 & 5 & $2 / 5$ & $15 p$ & 30 & $42 \times 10^{-16}$ \\
\hline 3 & $1 / 16$ & $29 \pi / 20$ & 38 & 10 & $2 / 5$ & $15 p$ & 90 & $6 \times 10^{-15}$ \\
\hline 4 & $-\pi / 120$ & $-\pi / 40$ & 40 & 64 & $2 / 3$ & 1 & $1 / 10$ & $-5 \times 10^{-15}$ \\
\hline 5 & $-\pi / 250$ & $-\pi / 100$ & 25 & 16 & 1 & $10 / 3$ & $7 / 5$ & $-7 \times 10^{-15}$ \\
\hline
\end{tabular}

Table 2: Input values for the superpotential parameters for three different cases. Case 2 has a vanishing one loop correction for $\phi_{2}$.

\begin{tabular}{|c|c|c|c|c|c|}
\hline & Case 1 & Case 2 & Case 3 & Case 4 & Case 5 \\
\hline$\langle s\rangle$ & 2.2 & 2.2 & 2.1 & 2.1 & 2.2 \\
\hline$\langle t\rangle$ & 1.2 & 1.1 & 1.6 & 1.1 & 1.1 \\
\hline$\langle\sigma\rangle$ & 1.0 & 1.0 & 1.0 & 0.0 & 0.0 \\
\hline$\left\langle\phi_{2}\right\rangle$ & 0.08 & 0.08 & 0.08 & 0.03 & 0.06 \\
\hline$F_{S}$ & $2.8 \times 10^{-16}$ & $1.3 \times 10^{-16}$ & $2.7 \times 10^{-16}$ & $1.1 \times 10^{-16}$ & $8.0 \times 10^{-17}$ \\
\hline$F_{T}$ & $-8.7 \times 10^{-15}$ & $-5.1 \times 10^{-15}$ & $-5.0 \times 10^{-15}$ & $6.7 \times 10^{-15}$ & $9.1 \times 10^{-15}$ \\
\hline$F_{\phi_{2}}$ & $-9.2 \times 10^{-17}$ & $-4.5 \times 10^{-17}$ & $-8.9 \times 10^{-17}$ & $1.3 \times 10^{-15}$ & $1.3 \times 10^{-15}$ \\
\hline$D_{A}$ & $4.4 \times 10^{-31}$ & $1.0 \times 10^{-32}$ & $5.9 \times 10^{-31}$ & $-3.8 \times 10^{-31}$ & $-4.8 \times 10^{-32}$ \\
\hline$D_{A} / m_{3 / 2}^{2}$ & 0.6 & 0.03 & 2.7 & -0.7 & -0.05 \\
\hline$V_{0} /\left(3 m_{3 / 2}^{2}\right)$ & -0.02 & -0.01 & -0.02 & -0.03 & -0.02 \\
\hline$m_{3 / 2}$ & $2.2 \mathrm{TeV}$ & $1.4 \mathrm{TeV}$ & $1.1 \mathrm{TeV}$ & $1.8 \mathrm{TeV}$ & $2.4 \mathrm{TeV}$ \\
\hline
\end{tabular}

Table 3: The values for field VEVs and soft SUSY breaking parameters at the minimum of the scalar potential. Note $F_{\Phi} \equiv \partial_{\Phi} \mathcal{W}+\left(\partial_{\Phi} \mathcal{K}\right) \mathcal{W}$.

is stabilized at $\sigma \approx 1$ in the racetrack cases 1,2 , and 3 . This value enforces a relative negative sign between the two terms dependent on $\operatorname{Re} T$. We plot the scalar potential $V$ in the Re $T$ direction for case $2\left(b, b_{2}>0\right)$ (Fig. 1(a) and for case $4\left(b, b_{2}<0\right)$ (Fig. 1(b)). Note the potential as a function of Re $S$ is qualitatively the same for both cases (Fig. 2).

At the meta-stable minimum of the scalar potential we find a vacuum energy which is slightly negative, i.e. of order $(-0.03$ to -0.01$) \times 3 m_{3 / 2}^{2} M_{P l}^{2}$ (see Table 3). Note, however, one loop radiative corrections to the vacuum energy are of order $\left(N_{T} m_{3 / 2}^{2} M_{S}^{2} / 16 \pi^{2}\right)$, where $N_{T}$ is the total number of chiral multiplets [83] and we have assumed a cut-off at the string scale $M_{S}$. With typical values $N_{T} \sim \mathscr{O}(300)$ and $M_{S} / M_{P l} \sim 0.1$, this can easily lift the 


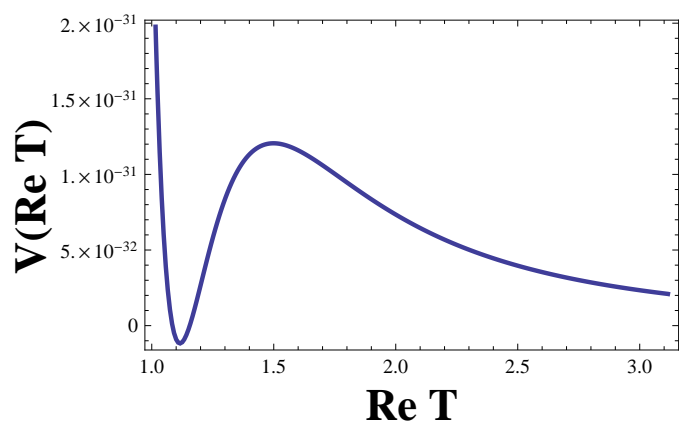

(a) The scalar potential in Case 2 for $\operatorname{Re} T$, with $b_{i}>0$.

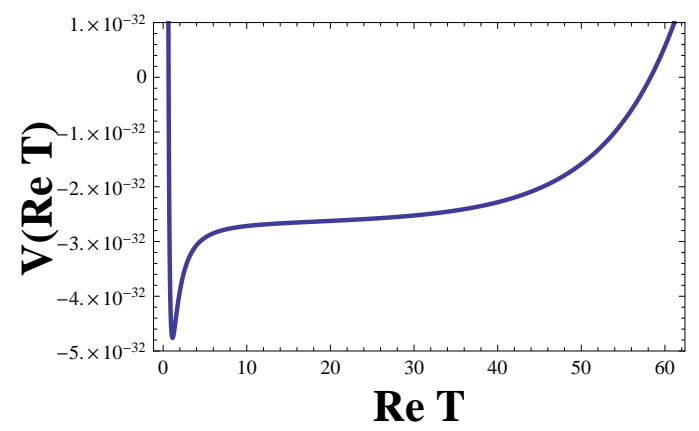

(b) The scalar potential in Case 4 for $\operatorname{Re} T$, with $b_{i}<0$.

Figure 1: As Re $T \rightarrow \infty$, the potential for $b_{i}>0$ mimics a Racetrack, which can be seen from Eqn. (37), for example. In the case where $b_{i}<0$, however, the potential exhibits a different asymptotic behavior. As $\operatorname{Re} T \rightarrow \infty$ the potential diverges, which means that theory is forced to be compactified [76, 82].

vacuum energy the rest of the way to give a small positive effective cosmological constant which is thus a meta-stable local dS minimum. Note that the constants $\lambda, R$ have also been used to adjust the value of the cosmological constant as well as, and more importantly for LHC phenomenology, the value of $D_{A}$ (see Fig. 3).

The two massless fields can be seen as the result of two $U(1)$ symmetries; the first is a $U(1)_{R}$ symmetry and the second is associated with the anomalous $U(1)_{A}$. The $U(1)_{R}$ is likely generic (but approximate), since even the "constant" superpotential term needed to obtain a small cosmological 


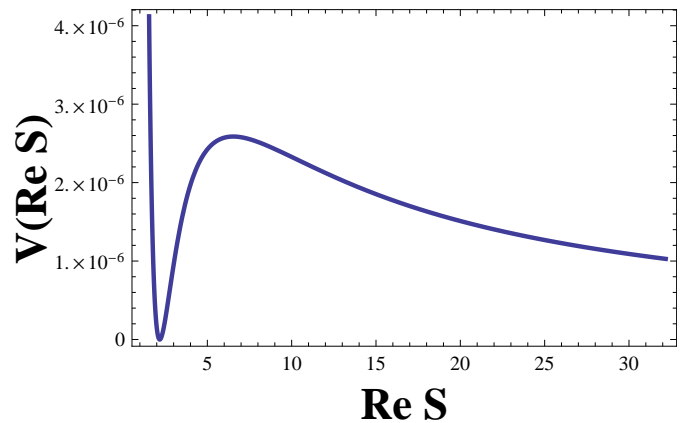

Figure 2: The scalar potential in the $\operatorname{Re} S$ direction for Case 2.

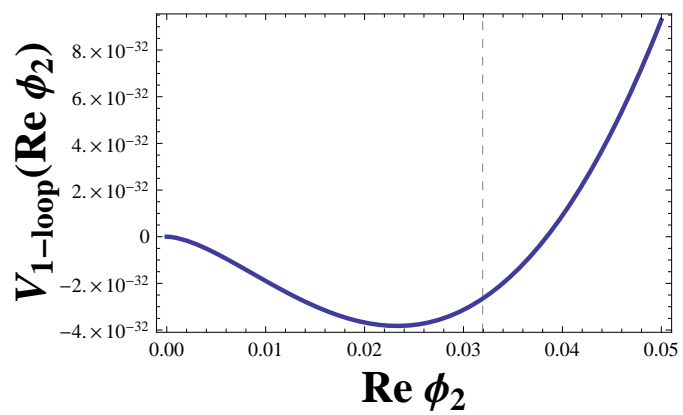

Figure 3: The one loop Coleman-Weinberg potential (Case 4) for $\phi_{2}$. The dashed line represents the VEV of $\phi_{2}$ in the minimum of the full potential.

constant necessarily comes with $\eta(T)$ moduli dependence. Since we have approximated $\eta(T) \sim \exp (-\pi T / 12)$ by the first term in the series expansion (Eqn. 17), the symmetry is exact. However higher order terms in the expansion necessarily break the $U(1)_{R}$ symmetry. The $U(1)_{A}$ symmetry is gauged.

One can express the fields $S, T$, and $\phi_{2}$ in the following basi:16:

$$
\begin{aligned}
S & \equiv s+i \sigma \\
T & \equiv t+i \tau \\
\phi_{2} & \equiv \varphi_{2} e^{i \theta_{2}} .
\end{aligned}
$$

The transformation properties of the fields $\sigma, \tau$ and $\theta_{2}$ under the two U(1)'s

\footnotetext{
${ }^{16}$ The fields $\chi$ and $\phi_{1}$ cannot be expressed in polar coordinates as they receive zero $\mathrm{VEV}$, and cannot be canonically normalized in this basis.
} 
are given by

$$
\begin{aligned}
& U(1)_{R}:\left\{\begin{array}{lll}
\tau & \rightarrow \tau+c \\
\sigma & \rightarrow \sigma+\frac{-b_{2}+b}{a} c
\end{array},\right. \\
& U(1)_{A}:\left\{\begin{array}{lll}
\theta & \rightarrow \theta-\frac{9}{r} c^{\prime} \\
\sigma & \rightarrow \sigma-\frac{9 p}{a \cdot r} c^{\prime}
\end{array},\right.
\end{aligned}
$$

where $c, c^{\prime}$ are arbitrary constants and for the definition of $r$ see footnote 9 . The corresponding Nambu-Goldstone (NG) bosons are given by

$$
\begin{aligned}
\chi_{\mathrm{NG}}^{1} & =\frac{a}{-b_{2}+b} \sigma+\tau, \\
\chi_{\mathrm{NG}}^{2} & =\tilde{N}\left(-\sigma+\frac{-b_{2}+b}{a} \tau\right)+\frac{1}{p} \theta_{2},
\end{aligned}
$$

where $\tilde{N}$ is a normalization factor. One can then calculate the mass matrix in the $\sigma-\tau-\theta_{2}$ basis and find two zero eigenvalues (as expected) and one non-zero eigenvalue. The two NG modes, in all cases, can be shown to be linear combinations of the two eigenvectors of the two massless states. The $\mathrm{U}(1)_{A} \mathrm{NG}$ boson is eaten by the $\mathrm{U}(1)_{A}$ gauge boson, while the $\mathrm{U}(1)_{R}$ pseudo-NG boson remains as an "invisible axion" [84]. The $U(1)_{R}$ symmetry is non-perturbatively broken (by world-sheet instantons) at a scale of order

$$
\left\langle e^{\mathcal{K} / 2} \mathcal{W} e^{-\pi T}\right\rangle \approx m_{3 / 2}\left\langle e^{-\pi T}\right\rangle \sim 0.02 m_{3 / 2}
$$

in Planck units, resulting in an "axion" mass of order $10 \mathrm{GeV}$ and decay constant of order $M_{P l} 17$

Before discussing the rest of the moduli, in a more complete string model, and how they would be stabilized or the LHC phenomenology of the miniversion of the mini-landscape models, it is worth comparing our analysis with some previous discussions in the literature.

In a series of two papers by Dvali and Pomarol [31, 32], the authors consider an anomalous U(1) with two charged singlet fields. The $D$ term is given by 18

$$
D_{A}=q\left|\phi_{+}\right|^{2}-\left|q_{-}\right|^{2}+\xi
$$

\footnotetext{
${ }^{17}$ In addition, the heterotic orbifold models might very well have the standard invisible axion 85 .

${ }^{18}$ We refer to the anomalous $U(1)$ as $U(1)_{A}$ and not $U(1)_{X}$, as in the papers referenced below.
} 
The gauge invariant superpotential is

$$
\mathcal{W}=m \phi_{+} \phi_{-},
$$

where $m$ has some charge under $\mathrm{U}(1)_{A}$. They suggest a few different ways to generate $m$. The first is with some high power of one of the $\phi$ fields:

$$
\mathcal{W} \sim \phi_{-}^{q} \phi_{+} \Rightarrow m \equiv\left\langle\phi_{-}\right\rangle^{q-1}
$$

The second is by giving the $\phi$ a coupling to some quarks from a SUSY QCD theory that becomes strongly coupled. The scale, $\Lambda_{S Q C D}$ then serves as the mass term in the superpotential. They do not, however, consider dilaton dependence, and their $D$ term is static, not dynamic. They also work in the global SUSY limit, so they do not consider up-lifting.

In a paper by Binetruy and Dudas [30], the authors assume that $S$ can be stabilized at some finite value $S_{0}$, possibly through some extra $S$ dependent term in the superpotential and they assume that $F_{S}\left(S_{0}\right)=0$. In their setup, they have an anomalous $\mathrm{U}(1)$, some charged singlets, and some hidden sector SQCD with matter. The singlets couple to matter, and SQCD becomes strongly coupled, generating a scale, just as in our analysis. Since they are working in the global SUSY limit, they are not concerned with up-lifting.

Lalak [33] considers several types of models with an anomalous U(1), some charged singlets, and some coupling to the dilaton $S$. In the last section, he considers superpotentials with an exponential dependence on $S$. He then assumes that $S_{0}$ is a (globally) supersymmetric minimum of the potential. Also, working in global SUSY, he does not address up-lifting.

In a paper by Dudas and Mambrini [36, the authors consider one modulus, one singlet field, and an $S U(N)$ with one flavor of quarks. The $S U(N)$ becomes strongly coupled, and the superpotential and Kähler potential look like:

$$
\begin{array}{r}
\mathcal{W}=w_{0}+\left(c / X^{2}\right) e^{-a T}+m \phi^{q} X \\
\mathcal{K}=-3 \log \left(T+\bar{T}-|X|^{2}-|\phi|^{2}\right)
\end{array}
$$

where $X$ is the meson field and $\phi$ is the singlet. Note, the modulus appearing in the exponent is $T$, not $S$. They find that the only consistent minimum with approximately zero cosmological constant requires $m_{3 / 2} \sim \xi$. So either the gravitino mass is of order the GUT scale or for the gravitino mass of order a TeV, the meson charge must satisfy $q \sim 10^{-8}$. 
In a paper by Dudas et al. 38, the authors consider a single modulus and two singlet fields:

$$
\begin{aligned}
D_{A} & =\left|\phi_{+}\right|^{2}-\left|\phi_{-}\right|^{2}+\xi \\
\mathcal{W} & =w_{0}+m \phi_{+} \phi_{-}+a \phi_{-}^{q} e^{-b T} .
\end{aligned}
$$

They do not discuss the origin of the constant $w_{0}$. They suggest that $m$ might come from non-perturbative effects. Note the latter is crucial, since $m$ affects the up-lifting of the scalar potential. They are also interested in large volume compactifications, as $t \equiv \operatorname{Re} T \approx 60$. Given their SUSY breaking scheme, they go on to look at the low energy spectrum. However, they neglect the $D$ term contributions to the soft masses, claiming that there are only two possibilities for the low energy physics:

- Because $\xi>0$, some SM quarks and leptons carry positive $\mathrm{U}(1)_{A}$ charges. This leads to scalar masses (for them) of around $100 \mathrm{TeV}$, and may give an unstable low energy spectrum.

- All SM quarks and leptons are neutral under $\mathrm{U}(1)_{X}$. This implies that there should be more matter that is charged under the MSSM and $\mathrm{U}(1)_{A}$.

It seems that they have missed an important possibility, namely that matter in the MSSM appears with $\mathrm{U}(1)_{A}$ charges of both signs. This actually seems to be the generic case, at least in the mini-landscape models.

The last paper we consider, by Gallego and Serone [39], contains an analysis which is possibly most similar to that in this paper. There are however two major differences. If one neglects all non-perturbative dependence on the dilaton and Kähler moduli, then their superpotential is of the form $\mathcal{W} \supset \phi^{q} \chi$ and the $\mathrm{D}$ term is given by $D_{A}=q|\chi|^{2}-|\phi|^{2}+\xi$. Hence the model does not have a supersymmetric minimum in the global limit, due to a conflict between $F_{\chi}=0$ and $D_{A}=0$. However in oure model (Eqn. 37) there is a supersymmetric solution when non-perturbative effects are ignored. Finally, the authors were not able to find a supersymmetry breaking solution, like ours, with just one hidden non-Abelian gauge sector.

As an aside, we note that Casas et al. [79] study a similar problem of moduli stabilization and SUSY breaking, but without the anomalous U(1). However, their model is very different from ours, but they do include the one loop Coleman-Weinberg corrections. 


\section{Moduli stabilization continued - the twisted sector and blow-up moduli}

In our discussion above we considered a simple model which is representative of heterotic orbifold models. Our simple model had only a few moduli, i.e. the dilaton, $S$, a volume modulus, $T$, and three chiral singlet 'moduli', $\chi, \phi_{1}, \phi_{2}$. Any heterotic orbifold construction, on the other hand, will have several volume and complex structure moduli and, of order 50 to 100 chiral singlet moduli. The superpotential for the chiral singlet moduli is obtained as a polynomial product of holomorphic gauge invariant monomials which typically contain hundreds of terms at each order (with the number of terms increasing with the order). In the "mini-landscape" analysis, supersymmetric vacua satisfying $F=D=0$ constraints to sixth order in chiral singlet moduli could be found. Although there are many flat directions in moduli space, the anomalous $D$-term fixes at least one holomorphic gauge invariant monomial to have a large value. Our simple model expressed this fact with the chiral singlets $\chi, \phi_{1}, \phi_{2}$, where the VEVs were fixed by the global SUSY minimum with $\left\langle\phi_{2}\right\rangle$ fixed by the $U(1)_{A} D$-term.

In addition to the non-Abelian hidden gauge sector considered in the simple model, a generic orbifold vacuum also has additional $U(1)$ gauge interactions and vector-like exotics which obtain mass proportional to chiral singlet VEVs. Some of these singlets are assumed to get large VEVs (of order the string scale). These are the ones giving mass to the extra $U(1)$ gauge sector and vector-like exotics. These same VEVs generate non-trivial Yukawa couplings for quarks and leptons. Moreover, there are chiral singlets which get zero VEVs, such as $\chi$ and $\phi_{1}$. For example, in the "mini-landscape" benchmark model 1, the electroweak Higgs $\mu$ term is zero in the supersymmetric limit. The question arises as to what happens to all these VEVs once supersymmetry is broken.

We now sketch the fact that the supersymmetry breaking discussed above, ensuing from $F$-terms, $F_{S}, F_{T}, F_{\phi_{2}} \neq 0$ and driven by the non-perturbative superpotential, inevitably leads to a stabilization of the many singlet 'moduli' of the heterotic orbifold vacuum. We shall consider here 3 classes of heterotic MSSM singlets. 


\subsection{Singlets with polynomial Yukawa couplings}

Let us first consider singlets having polynomial Yukawa couplings in the superpotential, which in case of a coupling arising among purely untwisted sector fields $\phi_{i}^{(U)}$ are perturbatively generated, and in the other case involving at least one twisted sector field $\phi_{i}^{(T)}$ are non-perturbatively generated (see Section 2.2). The latter case is actually the most common situation. Restricting again for reasons of simplicity to the case of a single scalar field of the type under consideration, we can describe the two cases as follows:

- i)

$$
\mathcal{K}=-3 \log \left(T+\bar{T}-\bar{\phi}^{(U)} \phi^{(U)}\right) \quad, \quad \mathcal{W} \supset \lambda \cdot\left(\phi^{(U)}\right)^{N}, N \geq 3
$$

Note that the untwisted sector scalar fields $\phi^{(U)}$, being inherited from the bulk 248 in 10d, appear this way in the Kähler potential.

- ii)

$$
\mathcal{K}=-3 \log (T+\bar{T})+c \bar{\phi}^{(T)} \phi^{(T)} \quad, \quad \mathcal{W} \supset e^{-b T}\left(\phi^{(T)}\right)^{N}, N \geq 3
$$

Here the exponential dependence on $T$ arises from the $\eta$-function, which a non-perturbatively generated Yukawa coupling must have for reasons of modular invariance (see Section 2.2).

- iii)

$$
\begin{gathered}
\mathcal{K}=-3 \log \left(T+\bar{T}-\bar{\phi}^{(U)} \phi^{(U)}\right)+c \bar{\phi}^{(T)} \phi^{(T)} \\
\mathcal{W} \supset \lambda e^{-b T}\left(\phi^{(T)}\right)^{N}+\tilde{\lambda} e^{-\tilde{b} T}\left(\phi^{(T)}\right)^{\tilde{N}}\left(\phi^{(U)}\right)^{M} \text { with } M, N, \tilde{N} \geq 2
\end{gathered}
$$

Here, too, the exponential dependence on $T$ from the $\eta$-function dependence of a non-perturbatively generated Yukawa coupling.

The calculation in case i) simplifies by the fact that there $K$ fulfills an extended no-scale relation

$$
\begin{aligned}
\mathcal{K}_{i} \mathcal{K}^{i \bar{j}} \mathcal{K}_{\bar{j}} & =3 \quad \forall i, j=T, \phi^{(U)} \\
\mathcal{K}^{i}=\mathcal{K}^{i \bar{j}} \mathcal{K}_{\bar{j}} & =-\mathcal{V} \cdot \delta_{T}^{i} \quad, \quad \mathcal{V} \equiv\left(T+\bar{T}-\bar{\phi}^{(U)} \phi^{(U)}\right)
\end{aligned}
$$


which implies for the F-term scalar potential a result

$$
\begin{aligned}
& V_{F}=e^{\mathcal{K}}\left[\mathcal{K}^{\phi^{(U)} \bar{\phi}^{(U)}}\left(\left|\partial_{\phi^{(U)}} \mathcal{W}\right|^{2}+\left(\partial_{\phi^{(U)}} \mathcal{W} \cdot \overline{\mathcal{K}_{\phi^{(U)}} \mathcal{W}}+\text { c.c. }\right)\right)\right. \\
& \left.+\frac{\mathcal{V}}{3}(T+\bar{T})\left|\partial_{T} \mathcal{W}\right|^{2}+\left(\mathcal{V} \partial_{T} \mathcal{W}+\text { c.c. }\right)\right] .
\end{aligned}
$$

It is clear then that one solution to $\partial_{\phi^{(U)}} V_{F}=0$ is given by

$$
\partial_{\phi^{(U)}} \mathcal{W}=\partial_{\phi^{(U)}} \mathcal{V}=0 \quad \Rightarrow \quad\left\langle\phi_{(U)}\right\rangle=0
$$

because $\partial_{\phi^{(U)}} \partial_{T} \mathcal{W} \equiv 0 \forall \phi^{(U)}$. This implies that those untwisted sector singlets that were stabilized at the origin in global supersymmetry by a purely untwisted sector Yukawa coupling remain so even in supergravity.

For the twisted sector case ii) we find the scalar potential to be

$$
\begin{aligned}
V_{F} & =e^{\mathcal{K}}\left[\mathcal{K}^{\phi^{(T)} \bar{\phi}^{(T)}}\left|D_{\phi^{(T)}} \mathcal{W}\right|^{2}+\mathcal{K}^{T \bar{T}}(\left|\partial_{T} \mathcal{W}\right|^{2}+\underbrace{\partial_{T} \mathcal{W}}_{\sim F_{T}} \overline{\mathcal{K}_{T} \mathcal{W}}+\text { c.c. })\right] \\
& \sim e^{-2 b T}\left(\bar{\phi}^{(T)} \phi^{(T)}\right)^{N-1}-F_{T}(T+\bar{T}) e^{-b T}\left(\phi^{(T)}\right)^{N}+\text { c.c. }
\end{aligned}
$$

which gives two solutions to $\partial_{\phi^{(T)}} V_{F}=0$ as

$$
\left\langle\phi^{(T)}\right\rangle=0 \quad \bigvee\left\langle\phi^{(T)}\right\rangle \sim\left(\frac{F_{T}(T+\bar{T})}{e^{-b T}}\right)^{\frac{1}{N-2}} \sim\left(\frac{m_{3 / 2}}{e^{-b T}}\right)^{\frac{1}{N-2}} .
$$

This implies that the $\phi^{(T)}$ get stabilized either at the origin, or at non-zero but small VEVs $\ll 1$. Their value in the latter case approaches $\phi^{(T)} \sim M_{\mathrm{GUT}}$ for non-perturbative Yukawa couplings of order $N \gtrsim 5$ and $m_{3 / 2} \sim \mathrm{TeV}$ (which can be interesting for phenomenological reasons involving heavy vector-like non-MSSM matter).

Finally, we note that case iii) reduces to case ii). To see this, note, that the structure of $\mathcal{K}$ and $\mathcal{W}$ given in case iii) does not the change the arguments given for case i) which implies that in case iii) we still find $\left\langle\phi_{(U)}\right\rangle=0$. This, however, immediately gives us

$$
\left.\mathcal{W}\right|_{\left\langle\phi_{(U)}\right\rangle=0} \supset \lambda e^{-b T}\left(\phi^{(T)}\right)^{N}
$$

which is case ii). 


\subsection{Singlet directions which are $F$ - and $D$-flat in global supersymmetry}

There are many directions in singlet field space in our heterotic constructions which are $F$ - and $D$-flat in global supersymmetry. Let us denote these fields by $-\phi_{i}^{(f)}$, and the remaining set of non-flat directions in field space by $\chi_{i}$. $D$ flatness entails that the $D$-terms do not depend on the $\phi_{i}^{(f)} . F$-flatness implies that $F_{\phi_{i}^{(f)}}=\partial_{\phi_{i}^{(f)}} \mathcal{W}\left(\phi_{i}^{(f)}, \chi_{i}\right)=$ const. for all values of $\left\langle\phi_{i}^{(f)}\right\rangle$. Generically this implies that $\left\langle\chi_{i}\right\rangle=0$.

Simplifying to the case of a single $\chi$, this leads to a consideration of 2 cases

- i)

$$
\begin{aligned}
F_{\phi_{i}^{(f)}} & =0 \quad \forall \phi_{i}^{(f)} \\
\Rightarrow \mathcal{W} & \supset e^{-b T} \chi \mathfrak{f}\left(\phi_{i}\right) \quad \bigvee \quad \mathcal{W} \supset e^{-b T} \chi^{p} \mathfrak{f}\left(\phi_{i}\right), p \geq 2
\end{aligned}
$$

- ii)

$$
\begin{aligned}
F_{\phi_{i}^{(f)}} & =\text { const. } \neq 0 \quad \forall \phi_{i}^{(f)} \\
\Rightarrow \mathcal{W} & \supset \lambda e^{-b T} f\left(\tilde{\phi}_{j}\right) \phi_{i}^{(f)}
\end{aligned}
$$

where the $\tilde{\phi}_{j}$ VEVs are assumed fixed by other terms in the superpotential and $\mathfrak{f}$ is an arbitrary function of its argument.

We consider first case i). At the supersymmetric minimum satisfying $\partial_{\chi} \mathcal{W}=\partial_{\phi_{i}} \mathcal{W}=0$, we have $\langle\chi\rangle=0$ with $\left\langle\phi_{i}\right\rangle$ arbitrary (subject, for the first case only, to the condition $\left.\mathfrak{f}\left(\phi_{i}\right)=0\right)$. In this example we have $\chi \in$ $\left\{\chi_{i}\right\}$ and $\phi_{i} \in\left\{\phi_{i}^{(f)}\right\}$. Note the fields $\phi_{i}^{(f)}$ effectively do not appear in the superpotential at its minimum.

We now argue that the fields $\phi_{i}^{(f)}$ are stabilized by the corrections from supergravity in the $F$-term scalar potential. Namely, consider for sake of simplicity the case of a single such field $\phi^{(f)}$ and $\chi$ with

$$
\begin{aligned}
\mathcal{K} & =-3 \log (T+\bar{T})+c \bar{\phi}^{(f)} \phi^{(f)}+c^{\prime} \bar{\chi} \chi \\
\partial_{\chi} \mathcal{W} & =\partial_{\phi^{(f)}} \mathcal{W} \equiv 0 \quad \text { for } \quad\langle\chi\rangle=0
\end{aligned}
$$


we get the F-term scalar potential in supergravity to be (for the twisted sector case ii) we find the scalar potential to be

$$
\begin{aligned}
V_{F}= & e^{\mathcal{K}}\left(\mathcal{K}^{\phi^{(f)} \bar{\phi}^{(f)}}\left|D_{\phi(f)} \mathcal{W}\right|^{2}+\mathcal{K}^{\chi \bar{\chi}}\left|D_{\chi} \mathcal{W}\right|^{2}+\mathcal{K}^{T \bar{T}}\left|D_{T} \mathcal{W}\right|^{2}-3|\mathcal{W}|^{2}\right) \\
= & e^{\mathcal{K}}\left(c \bar{\phi}^{(f)} \phi^{(f)}-\kappa\right) \cdot|\mathcal{W}|^{2} \\
\approx & |\mathcal{W}|^{2} \cdot\left[-c(\kappa-1) \bar{\phi}^{(f)} \phi^{(f)}-\frac{c^{2}(\kappa-2)}{2}\left(\bar{\phi}^{(f)} \phi^{(f)}\right)^{2}+\right. \\
& \left.\quad-\frac{c^{3}(\kappa-3)}{6}\left(\bar{\phi}^{(f)} \phi^{(f)}\right)^{3}+\frac{c^{4}(4-\kappa)}{24}\left(\bar{\phi}^{(f)} \phi^{(f)}\right)^{4}+\ldots\right]
\end{aligned}
$$

Note, we maintain $\langle\chi\rangle=0, \mathcal{W} \neq 0$ is due to other sectors of the theory and $\kappa=\left(3-\mathcal{K}^{T \bar{T}}\left|D_{T} \mathcal{W}\right|^{2} /|\mathcal{W}|^{2}\right) \leq 3$ is a positive semi-definite number of order 3. This scalar potential is unbounded from above at large field values, $\phi^{(f)}$, thus driving the VEV to large-field value. To this order in $V_{F}$ we find

$$
\left\langle\phi^{(f)}\right\rangle \sim \frac{1}{\sqrt{c}} .
$$

This implies that supergravity effects will serve to stabilize all the globally supersymmetric and $F$ - and $D$-flat singlet fields generically at large values of $\mathcal{O}(1)$. Note, that the non-perturbative effects coming from gauginocondensation in the hidden sector will add dependence of $\mathcal{W}$ on $\phi^{(f)}$ beyond the global mini-landscape analysis. This may render $\kappa$ a weak function of $\phi^{f}$ such that we may for some of the globally supersymmetric and $F$ - and $D$-flat fields $\phi^{(f)}$ have $\kappa<1$ at small $\phi^{(f)}$ while $1<\kappa<3$ at larger values of $\phi^{(f)}$. In this situation the involved $\phi^{(f)}$-type singlets will acquire vacua at both $\left\langle\phi^{(f)}\right\rangle=0$ and $\left\langle\phi^{(f)}\right\rangle \sim 1 / \sqrt{c}$. The $\chi$-like fields will have their VEVs near the origin, i.e. they may be shifted from the origin by small SUSY breaking effects.

Let us now turn to case ii) of $F$-flat but non-supersymmetric singlet directions and look for vacua stabilizing $\phi^{(f)} \ll 1$ using again

$$
\mathcal{K}=-3 \log (T+\bar{T})+\bar{\phi}^{(f)} \phi^{(f)}+\bar{\chi} \chi .
$$


The scalar potential is

$$
\begin{aligned}
V_{F}= & e^{\mathcal{K}}[\mathcal{K}^{T \bar{T}} \underbrace{\left\langle D_{T} \mathcal{W}\right\rangle}_{=F_{T}} \overline{\partial_{T} \mathcal{W}}+\text { c.c. }+\mathcal{K}^{\left.\phi^{(f)} \bar{\phi}^{(f)}\left|D_{\phi(f)} \mathcal{W}\right|^{2}\right]} \\
\sim & \left\{\mathcal{K}^{T \bar{T}} F_{T} \cdot b \lambda e^{-b T} f(\chi) \phi^{(f)}+\right.\text { c.c. } \\
& \left.+\mathcal{K}^{\phi^{(f)} \bar{\phi}^{(f)}}\left[\lambda e^{-b T} f(\chi)\left(1+\bar{\phi}^{(f)} \phi^{(f)}\right)+\bar{\phi}^{(f)}\langle\mathcal{W}\rangle\right]^{2}\right\} .
\end{aligned}
$$

In the desired regime of $\phi^{(f)} \ll 1$ this gives us two sub-cases:

- iia)

$$
\mathcal{K}^{\phi^{(f)} \bar{\phi}^{(f)}} F_{\phi^{(f)}} \ll \mathcal{K}^{T \bar{T}} F_{T}
$$

- iib)

$$
\mathcal{K}^{\phi^{(f)} \bar{\phi}^{(f)}} F_{\phi^{(f)}} \gg \mathcal{K}^{T \bar{T}} F_{T}
$$

In case iia) $\phi^{(f)} \ll 1$ implies that $F_{\phi^{(f)}} \equiv \lambda e^{-b T} f(\langle\chi\rangle) \ll\langle\mathcal{W}\rangle$ and thus $\partial_{\phi(f)} V_{F}=0$ gives us

$$
\left\langle\phi^{(f)}\right\rangle \sim \frac{\left\langle F_{\phi^{(f)}}\right\rangle}{\langle\mathcal{W}\rangle} \ll 1
$$

which is thus a self-consistent vacuum.

In the opposite situation we get $F_{\phi^{(f)}} \equiv \lambda e^{-b T} f(\langle\chi\rangle) \gg\langle\mathcal{W}\rangle,\left\langle F_{T}\right\rangle$. Using again $\phi^{(f)} \ll 1$ this leads to

$$
\left\langle\phi^{(f)}\right\rangle \sim \frac{\left\langle F_{T}\right\rangle}{\left\langle F_{\phi^{(f)}}\right\rangle} \ll 1 .
$$

Thus, even the $F$-flat but non-supersymmetric singlet directions of case ii) get stabilized by supersymmetry breaking effects from the bulk moduli stabilization at generically small but non-zero VEVs.

This property, of all $F$ - and $D$-flat singlet fields generically acquiring non-zero VEVs from supersymmetry breaking in the bulk moduli stabilizing sector through supergravity, dynamically ensures the decoupling of all vectorlike non-MSSM matter at low-energies as checked in global supersymmetry for the mini-landscape setup.

Note, that the overall vacuum structure of the F-flat singlet fields implicates a choice of initial conditions. The amount of non-MSSM vector-like 
extra matter in the mini-landscape constructions which decouples from low energies depends on the choice of the globally $F$-flat singlets $\phi_{i}^{(f)}$ placed at their non-zero VEV vacuum instead of their zero VEV vacuum. Thus, the choice of initial conditions in the vacuum distribution among the set of globally $F$-flat singlet fields characterizes how close to the MSSM one can get when starting from one of the mini-landscape models.

Assuming now that one finds successful eternal inflation occurring somewhere in the mini-landscape, this choice of initial conditions turns into a question of cosmological dynamics. In this situation, all possible initial conditions of the set of globally $F$-flat singlets were potentially realized in a larger multiverse. The choice of initial conditions on the singlets in the globally $F$-flat sector would then be amenable to anthropic arguments and might be eventually determined by selection effects.

\section{SUSY spectrum}

Now that we understand how SUSY is broken, we can calculate the spectrum of soft masses. The messenger of SUSY breaking is mostly gravity, however, there are other contributions from gauge and anomaly mediation.

\subsection{Contributions to the soft terms}

At tree level, the general soft terms for gravity mediation are given in References [90, 91, 92, 93, 94]. The models described in this paper contain an additional contribution from the $F$-term of a scalar field $\phi_{2}$. Following References [90, 91, 94], we define

$$
F^{I} \equiv e^{\mathcal{K} / 2} \mathcal{K}^{I \bar{J}}\left(\overline{\mathcal{W}}_{\bar{J}}+\overline{\mathcal{W}} \mathcal{K}_{\bar{J}}\right)
$$

\subsubsection{SUGRA effects}

Gaugino masses

The tree level gaugino masses are given by

$$
M_{a}^{(0)}=\frac{g_{a}^{2}}{2} F^{n} \partial_{n} f_{a}(S)=\frac{g_{a}^{2}}{2} F^{S} .
$$

At tree level, the gauge kinetic function in heterotic string theory is linear in the dilaton superfield $S$, and only dependent on the $T$ modulus at one loop. 
It is important to note the enhancement of $F^{S}$ relative to $F_{S}$ : naively, one might guess that loop corrections to the gaugino masses might be important, however

$$
F^{S}>>\frac{F^{T}}{16 \pi^{2}}
$$

thus loop corrections will be neglected.

A Terms

At tree level, the $A$ terms are given by

$$
A_{I J K}^{(0)}=F^{n} \partial_{n} \mathcal{K}+F^{n} \partial_{n} \log \frac{\mathcal{W}_{I J K}}{\kappa_{I} \kappa_{J} \kappa_{K}},
$$

where

$$
\mathcal{W}_{I J K} \equiv \frac{\partial^{3} \mathcal{W}}{\partial \Phi^{I} \partial \Phi^{J} \partial \Phi^{K}}
$$

and $\mathcal{K}$ is the Kähler potential. Neglecting $U$ dependence, we have

$$
\mathcal{K} \supset \Phi_{I} \bar{\Phi}^{I} \prod_{i}\left(T_{i}+\bar{T}_{i}\right)^{-n_{I}^{i}} \Rightarrow \kappa_{I} \equiv \prod_{i}\left(T_{i}+\bar{T}_{i}\right)^{-n_{I}^{i}}
$$

The $\kappa_{I}$ are the Kähler metrics for the chiral multiplets, $\Phi_{I}$, where as the $A$ terms are expressed in terms of canonically normalized fields. As before, the modular weights of the matter field are given by $n_{I}^{i}$.

In general, there are also tree level contributions to $A$ terms proportional to

$$
-\frac{F_{\phi_{2}}}{\left\langle\phi_{2}\right\rangle} \frac{\partial \log \mathcal{W}_{I J K}}{\partial \log \phi_{2}}
$$

These terms may be dominant, but unfortunately they are highly model dependent. They may give a significant contribution to $A_{b}$ and $A_{\tau}$, but in fact we find that the details of the low energy spectrum are not significantly effected.

\section{Scalar masses}

The tree level scalar masses are given by

$$
\left(M_{I}^{(0)}\right)^{2}=m_{3 / 2}^{2}-F^{n} \bar{F}^{\bar{m}} \partial_{n} \partial_{\bar{m}} \log \kappa_{I}+g_{G}^{2} f q_{A}^{I}\left\langle D_{A}\right\rangle \kappa_{I},
$$

where $g_{G}^{2}=1 / \operatorname{Re} S_{0}$ and we have implicitly assumed that the Kähler metric is diagonal in the matter fields. The factor $f$ re-scales the $U(1)_{A}$ charges 
$q_{A}$ from the mini-landscape "benchmark" model 1 [9], so they are consistent with the charges $q_{A}^{\prime}$ in our mini-version of the mini-landscape model. We have $q_{A}^{\prime}=q_{A} f=q_{A} \frac{48 \pi^{2}}{\operatorname{Tr} Q} \delta_{G S}$ with $\delta_{G S}=\frac{N_{f}}{4 \pi^{2}}$ (Eqn. 7) and $\operatorname{Tr} Q=\frac{296}{3}$ (Eqn. E.5, [9]) such that $\frac{\operatorname{Tr}\left(q^{\prime}\right)}{4 \pi^{2}}=\delta_{G S}$.

Again neglecting $U$ dependence, the Kähler metric for the matter fields depends only on the $T$ moduli, and we find

$$
\left(M_{I}^{(0)}\right)^{2}=m_{3 / 2}^{2}-\sum_{i} \frac{n_{I}^{i}\left|F^{T_{i}}\right|^{2}}{\left(T_{i}+\bar{T}_{i}\right)^{2}}+g_{G}^{2} f q_{A}^{I}\left\langle D_{A}\right\rangle /\left(2 \operatorname{Re} T_{0}\right)^{n_{I}^{3}}
$$

$\underline{\mu \text { and } B \mu \text { terms }}$

The $\mu$ term can come from two different sources:

$$
\mathcal{K} \supset Z\left(T_{i}+\bar{T}_{i}, U_{j}+\bar{U}_{j}, \ldots\right) \mathbf{H}^{u} \mathbf{H}^{d}, \mathcal{W} \supset \tilde{\mu}\left(\mathbf{s}_{I}, T_{i}, U_{j}, \ldots\right) \mathbf{H}^{u} \mathbf{H}^{d}
$$

In the orbifold models, Kähler corrections have not been computed, so the function $Z$ is a priori unknown. Such a term could contribute to the GiudiceMasiero mechanism [98]. When both $\tilde{\mu}$ and $Z$ vanish, the SUGRA contribution to the $\mu / B \mu$ terms vanish. On the other hand, in the class of models which we consider, we know that vacuum configurations exist such that $\tilde{\mu}=0$ to a very high order in singlet fields. Moreover $\tilde{\mu} \propto\langle\mathcal{W}\rangle$ which vanishes in the supersymmetric limit, but obtains a value $w_{0}$ at higher order in powers of chiral singlets. If $\mu$ is generated in this way, there is also likely to be a Peccei-Quinn axion [99, 100]. Finally, supergravity effects will also generate a $B \mu$ term.

\section{Loop corrections}

Finally, one can consider loop corrections to the tree level expressions in [90, 91, 94. This was done in References [95, 96], where the complete structure of the soft terms (at one loop) for a generic (heterotic) string model were computed in the effective supergravity limit. We have applied the results of [95, 96] to our models and find, at most, around a $10 \%$ correction to the tree level results of [90, 91, 94], 19]

\footnotetext{
${ }^{19}$ In estimating this result, we have assumed that the mass terms of the Pauli-Villars fields do not depend on the SUSY breaking singlet field $\phi_{2}$, and that the modular weights of the Pauli-Villars fields obey specific properties.
} 


\subsubsection{Gauge mediation}

The "mini-landscape" models generically contain vector-like exotics in the spectrum. Moreover it was shown that such states were necessary for gauge coupling unification [97. The vector-like exotics obtain mass in the supersymmetric limit by coupling to scalar moduli, thus they may couple to the SUSY breaking field $\phi_{2}$. We will consider the following light exotics to have couplings linear in the field $\phi_{2}$ :

$$
n_{3} \times(\mathbf{3}, 1)_{1 / 3}+n_{2} \times(1, \mathbf{2})_{0}+n_{1} \times(1,1)_{-1}+\text { h.c. }
$$

where the constants $n_{i}$ denote the multiplicity of states and (see Table 7 of Reference [97])

$$
n_{3} \leq 4 \text { and } n_{2} \leq 3 \text { and } n_{1} \leq 7 .
$$

The gauge mediated contributions split the gaugino masses by an amount proportional to the gauge coupling:

$$
\begin{aligned}
\left.M_{3}^{(1)}\right|_{\text {gmsb }} & =n_{3} \frac{g_{3}^{2}}{16 \pi^{2}} \frac{F^{\phi_{2}}}{\left\langle\phi_{2}\right\rangle}, \\
\left.M_{2}^{(1)}\right|_{\text {gmsb }} & =n_{2} \frac{g_{2}^{2}}{16 \pi^{2}} \frac{F^{\phi_{2}}}{\left\langle\phi_{2}\right\rangle}, \\
\left.M_{1}^{(1)}\right|_{\text {gmsb }} & =\frac{n_{3}+3 n_{1}}{10} \frac{g_{1}^{2}}{16 \pi^{2}} \frac{F^{\phi_{2}}}{\left\langle\phi_{2}\right\rangle} .
\end{aligned}
$$

It is interesting to note that this becomes more important as $\left\langle\phi_{2}\right\rangle$ decreases $/ F^{\phi_{2}}$ increases, or if there are a large number of exotics present.

The scalar masses in gauge mediation come in at two loops, and receive corrections proportional to

$$
\left.\left(M_{I}\right)^{2}\right|_{\mathrm{gmsb}} \sim\left(\frac{1}{16 \pi^{2}}\right)^{2}\left(\frac{F_{\phi_{2}}}{\phi_{2}}\right)^{2} .
$$

Unlike in the case of the gaugino masses, however, the tree level scalar masses are set by the gravitino mass. Typically

$$
16 \pi^{2} m_{3 / 2}>>\frac{F_{\phi_{2}}}{\phi_{2}}
$$

and the gauge mediation contribution gives about a $10 \%$ correction to the scalar masses, in our case. We will neglect their contributions in the calculation of the soft masses below. 


\subsection{Calculation of the soft terms - relevant details from the "mini-landscape"}

Given the relative sizes of the $F$-terms in the SUSY breaking sectors described in this paper, it is very difficult to make model-independent statements. This stems from the fact that $F^{T}$ plays a dominant role in the SUSY breaking. Because the Kähler metrics for the matter fields have generally different dependences on the $T$ modulus, the dependence of the soft terms on $F^{T}$ is typically non-universal. Moreover, the couplings of the SUSY breaking singlet field $\phi_{2}$ will necessarily depend on the details of a specific model. Thus, in order to make any statements about the phenomenology of these models, we will have to make some assumptions. With the general features of the "mini-landscape" models in mind, we will make the following assumptions:

1. SUSY breaking is dominated by $F_{\phi_{2}} \neq 0, F_{T_{3}} \neq 0, F_{S} \neq 0$. All other $F$ terms, including those due to the other $T$ and $U$ moduli, are subdominant;

2. the massless spectrum below $M_{\mathrm{S}}$ contains some vector-like exotics;

3. the untwisted sector contains the following Higgs and (3rd generation) matter multiplets: $\mathbf{H}_{u}, \mathbf{H}_{d}, \mathbf{Q}_{3}, \mathbf{U}_{3}^{c}, \mathbf{E}_{3}^{c}$;

4. the first two families have the same modular weights, see Table 4:

5. the SUSY breaking field, $\phi_{2}$, lives in the untwisted, or second or fourth twisted sector, with a modular weight given by $n^{3}=0$; and

6. we neglect possible $\phi_{2}$ dependence of the effective Yukawa terms.

Let us examine these assumptions in some more detail.

In general, gauge coupling unification in the "mini-landscape" models seems to require the existence of light vector-like exotics [97, whose masses can be as small as $\mathscr{O}\left(10^{9} \mathrm{GeV}\right)$. We further assume that these exotics couple to the SUSY breaking field $\phi_{2}$, giving a gauge mediated contribution to the gaugino masses above. We will make this contribution to the soft terms explicit in what follows. In assumption 2 we have specialized to the case where only "brane-localized" exotics are present in the model. These are states which come from the first and third twisted sectors of the model, and we refer the reader to [9, 97] for more details. 
The top quarks and the up Higgses live in the bulk and the string selection rules allow for the following coupling in the superpotential:

$$
\mathcal{W} \supset c \mathbf{Q}_{3} \mathbf{H}_{u} \mathbf{U}_{3}^{c} \text {. }
$$

The coupling $c$ is a pure number of $\mathscr{O}(1)$, and is free of any dependence on the moduli. The down and lepton Yukawas are a bit more involved, as they arise at a higher order in the stringy superpotential. We will take them to be of the following form:

$$
\mathcal{W} \supset \eta\left(T_{1}\right)^{p_{1}} \eta\left(T_{2}\right)^{p_{2}} \eta\left(T_{3}\right)^{p_{3}}\left(f_{1}\left(\left\langle\mathbf{s}_{I}^{5}\right\rangle\right) \mathbf{Q}_{3} \mathbf{H}_{d} \mathbf{D}_{3}^{c}+f_{2}\left(\left\langle\mathbf{s}_{I}^{5}\right\rangle\right) \mathbf{L}_{3} \mathbf{H}_{d} \mathbf{E}_{3}^{c}\right) .
$$

The $\mathbf{s}_{I}$ are other singlet fields in the model (excluding the SUSY breaking singlet field, $\phi_{2}$, as per our assumptions), and the numbers $p_{1}, p_{2}$ and $p_{3}$ are calculable in principle, given knowledge of the modular weights of the $\mathbf{s}_{I}$. As one might expect, the expressions for the $A$ terms explicitly depend on the value of $p_{3}$ in such a way that changing its value may result in a significant change in $A_{b}$ and $A_{\tau}$ at the string scale. The impact on the weak scale observables is much less severe, however, giving a correction of a few percent to the gaugino masses, and leaving the squark and slepton masses virtually unchanged. Motivated by the modular weight assignments in Table 4 , we will choose $p_{3}=0$. Note this choice gives us universal $A$ terms for the third generation.

One of the nice features of the "mini-landscape" models is the incorporation of a discrete $\left(D_{4}\right)$ symmetry between the first two families in the low energy effective field theory. Because of this symmetry, we expect the modular weights of these matter states to be the same [101], see Table 4. This will turn out to be very beneficial in alleviating the flavor problems that are generic in gravity mediated models of SUSY breaking: the scalar masses (at tree level) are given by a universal contribution (the gravitino mass squared) plus a contribution proportional to the modular weight. If the modular weights are the same between the first two generations, then the leading order prediction is for degenerate squark and slepton masses in the two light generations. Other contributions to the scalar masses come from gauge mediation and anomaly mediation, which do not introduce any new flavor problems into the low energy physics. 


\begin{tabular}{|c|c|c|}
\hline MSSM particle & Modular Weight $\vec{n}$ & $U(1)_{A}$ charge \\
\hline $\mathbf{Q}_{3}$ & $(0,1,0)$ & $4 / 3$ \\
$\mathbf{U}_{3}^{c}$ & $(1,0,0)$ & $2 / 3$ \\
$\mathbf{D}_{3}^{c}$ & $\left(\frac{1}{3}, \frac{2}{3}, 0\right)$ & $8 / 9$ \\
$\mathbf{L}_{3}$ & $\left(\frac{2}{3}, \frac{1}{3}, 0\right)$ & $4 / 9$ \\
$\mathbf{E}_{3}^{c}$ & $(1,0,0)$ & $2 / 3$ \\
first two gen. & $\left(\frac{5}{6}, \frac{2}{3}, \frac{1}{2}\right)$ & $7 / 18 \quad(\mathbf{1 0})$ \\
$\mathbf{H}_{u}$ & $(0,0,1)$ & $-5 / 18(\overline{\mathbf{5}})$ \\
$\mathbf{H}_{d}$ & $(0,0,1)$ & -2 \\
\hline \hline
\end{tabular}

Table 4: Modular weights of the MSSM states in the "mini-landscape" benchmark model 1A. For the first two generations, the $U(1)_{A}$ charges differ depending on whether the particle is in the $\mathbf{1 0}$ or $\overline{\mathbf{5}}$ of $S U(5)$. See [9] for details.

\subsection{Hierarchy of $F$-terms}

Note, in Section 3, we find (roughly)

$$
F_{T}>>F_{S} \gtrsim F_{\phi_{2}},
$$

for Cases 1, 2 and 3; and

$$
F_{T} \gtrsim F_{\phi_{2}}>>F_{S}
$$

for Cases 4 and 5, where

$$
F_{I} \equiv \mathcal{W}_{I}+\mathcal{W K}_{I}
$$

When one includes the relevant factors of the Kähler metric, we have (Table 51)

$$
F^{T}>F^{S}>>F^{\phi_{2}}
$$

for Cases 1, 2 and 3; and

$$
F^{T}>>F^{S} \sim F^{\phi_{2}}
$$

for Cases 4 and 5. $F^{S}$ is enhanced by a factor of $\mathcal{K}^{S \bar{S}} \sim(2+2)^{2}$, while $F^{\phi_{2}}$ is decreased by a factor of $\mathcal{K}^{\phi_{2} \bar{\phi}_{2}} \sim(2)^{-1 / 2} 20$ This means that although

\footnotetext{
${ }^{20}$ This is due to the assumed modular weight of the field $\phi_{2}$ (assumption 5 in Section 5.2).
} 


\begin{tabular}{|c|c|c|c|c|c|}
\hline & Case 1 & Case 2 & Case 3 & Case 4 & Case 5 \\
\hline$F^{S}$ & $6.6 \times 10^{-16}$ & $3.7 \times 10^{-16}$ & $4.2 \times 10^{-16}$ & $2.7 \times 10^{-16}$ & $2.1 \times 10^{-16}$ \\
\hline$F^{T}$ & $-2.2 \times 10^{-15}$ & $-1.2 \times 10^{-15}$ & $-1.4 \times 10^{-15}$ & $1.6 \times 10^{-15}$ & $2.2 \times 10^{-15}$ \\
\hline$F^{\phi_{2}}$ & $-1.1 \times 10^{-17}$ & $-6.5 \times 10^{-18}$ & $-7.7 \times 10^{-18}$ & $1.9 \times 10^{-16}$ & $1.8 \times 10^{-16}$ \\
\hline
\end{tabular}

Table 5: The hierarchy of $F$ terms in the five examples of the single condensate model we studied. Note that $F^{\Phi}$ is defined in Eqn. (68). All of the $F$ terms contribute to the soft masses, as they are all within an order of magnitude.

the singlet field $\phi_{2}$ was a dominant source of SUSY breaking, it is the least important when computing the soft terms, given the one condensate hidden sector of the known "mini-landscape" models studied in Section $3{ }^{21}$ Taking the details of the "mini-landscape" models into account, the soft terms at the string scale are given in Table 6 .

In the five chosen Cases, 2, 3 and 4 have a gravitino mass less than $2 \mathrm{TeV}$. The value of the gravitino mass can be adjusted by varying $w_{0}$. For Cases, 1, 3 (4) the Higgs up (down) mass squared is negative. This is a direct result of the sign of $D_{A}$ and the $U(1)_{A}$ charge of the Higgs' (see Table 4 for the $U(1)_{A}$ charges of all the MSSM states) 22 Note, the first and second generation squarks and sleptons are lighter than the third generation states at the string scale. This is a consequence of the significant $T$ modulus contribution to the first and second generation squark and slepton masses, due to their modular weights, Table 4. Finally we have included the possible

\footnotetext{
${ }^{21}$ In racetrack models $F_{S}$ is suppressed by more than an order of magnitude. In these cases $F_{\phi_{2}}$ is dominant 39 .

${ }^{22}$ Note, it is well known that the $D$-term VEV in supergravity is of order $\left\langle F^{i}\right\rangle^{2}$ [102, 93 . It is given by the relation$$
\left\langle D_{A}\right\rangle=2 M_{A}^{-2}\left\langle F^{i}\right\rangle\left\langle F_{j}^{*}\right\rangle\left\langle\partial_{i} \partial^{j} D_{A}\right\rangle .
$$

Thus the $D$-term contribution to the vacuum energy is negligible, but its contribution to scalar masses can be significant. Since $\left|F^{S}\right|^{2}<\left|F^{T}\right|^{2}, F^{T}$ is dominant in the above relation. However, the Kähler metric of $\phi_{2}$ which spontaneously breaks $U(1)_{A}$, in our case, does not include $T$, i.e. $\left\langle\left(\partial_{T} \partial^{T} D_{A}\right)\right\rangle=0$. Hence $\left\langle D_{A}\right\rangle$ is suppressed compared with $\left|F^{T}\right|^{2} / M_{P l}^{2}$, i.e. $\left\langle D_{A}\right\rangle:\left|F^{T}\right|^{2} / M_{P l}^{2}=\left|F^{S}\right|^{2}:\left|F^{T}\right|^{2}$ where we used $\left\langle\left(\partial_{S} \partial^{S} D_{A}\right)\right\rangle=\left(M_{A} / M_{P l}\right)^{2}$, because of the S-dependent FI term. We thank T. Kobayashi, private communication, for this analysis. However, it should be clear that we have also used the freedom available in the Coleman-Weinberg one-loop correction to further adjust the value of the $D$-term.
} 


\begin{tabular}{|c|c|c|c|c|c|c|c|c|c|c|}
\hline & \multicolumn{10}{|c|}{ All Masses in GeV } \\
\hline Parameter & \multicolumn{2}{|c|}{ Case 1} & \multicolumn{2}{|c|}{ Case 2} & \multicolumn{2}{|c|}{ Case 3} & \multicolumn{2}{|c|}{ Case 4} & \multicolumn{2}{|c|}{ Case 5} \\
\hline$m_{3 / 2}$ & \multicolumn{2}{|c|}{2159} & \multicolumn{2}{|c|}{1350} & \multicolumn{2}{|c|}{1133} & \multicolumn{2}{|c|}{1808} & \multicolumn{2}{|c|}{2375} \\
\hline$m_{H_{u}}$ & \multirow{2}{*}{\multicolumn{2}{|c|}{$\begin{array}{l}478 i \\
679\end{array}$}} & \multirow{2}{*}{\multicolumn{2}{|c|}{$\begin{array}{l}168 \\
216\end{array}$}} & \multirow{2}{*}{\multicolumn{2}{|c|}{$\begin{array}{c}372 i \\
495\end{array}$}} & \multirow{2}{*}{\multicolumn{2}{|c|}{$\begin{array}{c}688 \\
476 i\end{array}$}} & \multirow{2}{*}{\multicolumn{2}{|c|}{$\begin{array}{l}384 \\
251\end{array}$}} \\
\hline$m_{H_{d}}$ & & & & & & & & & & \\
\hline$M_{1}$ & \multirow{3}{*}{\multicolumn{2}{|c|}{$\begin{array}{c}362-0.3 n_{1}-0.1 n_{3} \\
362-1 n_{2} \\
362-1 n_{3} \\
\end{array}$}} & \multirow{3}{*}{\multicolumn{2}{|c|}{$\begin{array}{c}206-0.2 n_{1}-0.1 n_{3} \\
206+1 n_{2} \\
206+1 n_{3} \\
\end{array}$}} & \multirow{3}{*}{\multicolumn{2}{|c|}{$\begin{array}{c}243-0.2 n_{1}-0.1 n_{3} \\
243-1 n_{2} \\
243-1 n_{3} \\
\end{array}$}} & \multirow{3}{*}{\multicolumn{2}{|c|}{$\begin{array}{c}158+13 n_{1}+4 n_{3} \\
158+45 n_{2} \\
158+45 n_{3} \\
\end{array}$}} & \multirow{3}{*}{\multicolumn{2}{|c|}{$\begin{array}{c}118+7 n_{1}+2 n_{3} \\
118+23 n_{2} \\
118+23 n_{3} \\
\end{array}$}} \\
\hline$M_{2}$ & & & & & & & & & & \\
\hline$M_{3}$ & & & & & & & & & & \\
\hline$A_{t}$ & \multicolumn{2}{|c|}{3901} & \multicolumn{2}{|c|}{2466} & \multicolumn{2}{|c|}{1974} & \multicolumn{2}{|c|}{-3690} & \multicolumn{2}{|c|}{-4798} \\
\hline$A_{b}$ & \multicolumn{2}{|c|}{3901} & \multicolumn{2}{|c|}{2466} & \multicolumn{2}{|c|}{1974} & \multicolumn{2}{|c|}{-3690} & \multicolumn{2}{|c|}{-4798} \\
\hline$A_{\tau}$ & \multicolumn{2}{|c|}{3901} & \multicolumn{2}{|c|}{2466} & \multicolumn{2}{|c|}{1974} & \multicolumn{2}{|c|}{-3690} & -47 & \\
\hline & Gen. 1,2 & Gen. 3 & Gen. 1,2 & Gen. 3 & Gen. 1,2 & Gen. 3 & Gen. 1,2 & Gen. 3 & Gen. 1,2 & Gen. 3 \\
\hline$m_{\tilde{q}}$ & 1580 & 2288 & 966 & 1355 & 895 & 1446 & 1262 & 1657 & 1691 & 2361 \\
\hline$m_{\tilde{u}^{c}}$ & 1580 & 2225 & 966 & 1353 & 895 & 1299 & 1262 & 1734 & 1691 & 2368 \\
\hline$m_{\tilde{d}^{c}}$ & 1521 & 2246 & 964 & 1354 & 757 & 1350 & 1330 & 1709 & 1697 & 2366 \\
\hline$m_{\tilde{\ell}}$ & 1580 & 2203 & 964 & 1352 & 757 & 1246 & 1330 & 1759 & 1697 & 2370 \\
\hline$m_{\tilde{e}^{c}}$ & 1580 & 2225 & 966 & 1353 & 895 & 1299 & 1262 & 1734 & 1691 & 2368 \\
\hline
\end{tabular}

Table 6: Boundary conditions at the string scale. $n_{3}, n_{2}, n_{1}$ refer to possible intermediate mass vector-like exotics which couple to the SUSY breaking field $\phi_{2}$, see Eqn. (178).

gauge mediated SUSY breaking contribution to the gaugino masses, Table 6. This contribution is only significant for Cases 4 and 5, due to the larger value of $F_{\phi_{2}}$ in these cases.

\subsection{Weak scale observables}

We do not intend this work to be a comprehensive study of the parameter space of these models, so we will limit our weak scale analysis to the five cases studied in the single condensate model presented in this paper. The points are chosen subject to the following constraints:

- $\left.m_{h^{0}}\right|_{\mathrm{LEP}} \gtrsim 114.4 \mathrm{GeV}$

- successful electroweak symmetry breaking,

- $m_{\tilde{\chi}^{ \pm}} \gtrsim 94 \mathrm{GeV}$, and

- the low energy spectrum is free of tachyons. 
Note that we take $\operatorname{sgn}(\mu)>0$ and vary $\tan \beta$, and the number, $n_{i}$, of "messenger" exotics. We stay in the region of small to moderate $\tan \beta$ as the "mini-landscape" models do not tend to predict unification of the third family Yukawas. This can be seen from Eqns. (85) and (866), for example.

Using SoftSUSY (v3.1) [103], we preformed the RGE running from the string scale to the weak scale. We use the current value of the top quark mass [104]

$$
\left.m_{\text {top }}\right|_{\text {world avg. }}=173.1 \mathrm{GeV}
$$

and the strong coupling constant at $M_{\mathrm{Z}}$ [105]

$$
\alpha_{s}\left(M_{\mathrm{Z}}\right)=0.1176 \text {. }
$$

The $\mu$ parameter is obtained under the requirement of radiative electroweak symmetry breaking, and is of order the gravitino mass, as expected. This implies a fine tuning of order

$$
\frac{M_{\mathrm{Z}}^{2}}{m_{3 / 2}^{2}} \sim \mathscr{O}\left(10^{-2}\right) \text { to } \mathscr{O}\left(10^{-4}\right) .
$$

The results obtained from SoftSUSY are presented in Table 7. In this analysis, we have not included any possible gauge mediated SUSY breaking contributions. This assumes that all the vector-like exotics have mass at the string scale. In Case 2 and 3 we have the smallest gravitino masses, so the lightest SUSY partners. $\tan \beta=25$ in order for the light Higgs mass to be above the LEP bound. Note we assume a $\pm 2 \mathrm{GeV}$ theoretical uncertainty in the Higgs mass. In all 5 cases the Higgs mass is between the LEP bound and $121 \mathrm{GeV}$. All other Higgs masses are of order the gravitino mass. In all 5 cases the gluino mass is less than $1 \mathrm{TeV}$ and of order $600 \mathrm{GeV}$ or less in Cases 2, .. , 5. Thus the gluino is very observable at the LHC. In all cases, the lightest MSSM particle is the lightest neutralino. The next-to-lightest neutralino and the lightest chargino are approximately degenerate with mass of order twice the lightest neutralino mass. In Cases 2, 3 and 4 the lightest stop has mass less than $1 \mathrm{TeV}$. In Cases 2 and 4, the lightest stop is also the lightest squark. Thus in these cases the gluino will predominantly decay into a top - anti-top pair with missing energy (and possibly two energetic leptons). In Case 3, the lightest down squarks of the first two families are lighter than the lightest stop. In these cases gluinos will decay significantly into two light quark jets plus missing energy (and possibly two energetic leptons). 


\begin{tabular}{|c|c|c|c|c|c|c|c|c|c|c|c|}
\hline & & \multicolumn{10}{|c|}{ All Masses in $\mathrm{GeV}$ (defined at $M_{\mathrm{W}} \approx 80 \mathrm{GeV}$, unless otherwise noted.) } \\
\hline & Observable & \multicolumn{2}{|c|}{ Case 1} & \multicolumn{2}{|c|}{ Case 2} & \multicolumn{2}{|c|}{ Case 3} & \multicolumn{2}{|c|}{ Case 4} & \multicolumn{2}{|c|}{ Case 5} \\
\hline \multirow{4}{*}{ 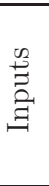 } & $m_{3 / 2}$ & \multicolumn{2}{|c|}{2159} & \multicolumn{2}{|c|}{1350} & \multicolumn{2}{|c|}{1133} & \multicolumn{2}{|c|}{1808} & \multicolumn{2}{|c|}{2375} \\
\hline & $\tan \beta$ & \multicolumn{2}{|c|}{10} & \multicolumn{2}{|c|}{25} & \multicolumn{2}{|c|}{25} & \multicolumn{2}{|c|}{10} & \multicolumn{2}{|c|}{4} \\
\hline & $\operatorname{sgn}(\mu)$ & \multicolumn{2}{|c|}{+} & \multicolumn{2}{|c|}{+} & \multicolumn{2}{|c|}{+} & \multicolumn{2}{|c|}{+} & \multicolumn{2}{|c|}{+} \\
\hline & $n_{1}, n_{2}, n_{3}$ & \multicolumn{2}{|c|}{$0,0,0$} & \multicolumn{2}{|c|}{$0,0,0$} & \multicolumn{2}{|c|}{$0,0,0$} & \multicolumn{2}{|c|}{$0,0,0$} & 0,0 & \\
\hline & $\mu\left(M_{\text {SUSY }}\right)$ & 222 & & 131 & & 13 & & 18 & & 26 & \\
\hline$\varphi$ & $m_{h^{0}}$ & 115 & & 113 & & 113 & & 121 & & 116 & \\
\hline 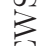 & $m_{H^{0}}$ & 229 & & 116 & & 130 & & 173 & & 27 & \\
\hline & $m_{A^{0}}$ & 230 & & 117 & & 13 & & $17^{\circ}$ & & 27 & \\
\hline & $m_{H^{+}}$ & 230 & & 117 & & 13 & & 173 & & 27 & \\
\hline th & $M_{1}$ & 15 & & 83 & & 10 & & 68 & & 5 & \\
\hline$\sum_{n}^{\infty}$ & $M_{2}$ & 27 & & 15 & & 18 & & 12 & & 10 & \\
\hline 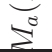 & $M_{3}$ & 77 & & 45 & & 53 & & 37 & & 27 & \\
\hline 10 & $\overline{m_{\tilde{g}}}$ & 91 & & 54 & & 63 & & 45 & & 36 & \\
\hline & $m_{\tilde{\chi}_{1}^{0}}$ & 15 & & 83 & & 9 & & 68 & & 5 & \\
\hline$\dot{0}$ & $m_{\tilde{\chi}_{2}^{0}}$ & 29 & & 16 & & 19 & & 13 & & 10 & \\
\hline ฮ & $m_{\tilde{\chi}_{3}^{0}}$ & -22 & & -13 & & -13 & & -18 & & -26 & \\
\hline$\dot{\dot{z}}$ & $m_{\tilde{\chi}_{4}^{0}}$ & 220 & & 130 & & 13 & & $18:$ & & 26 & \\
\hline$\stackrel{0}{z}$ & $m_{\tilde{\chi}_{1}^{ \pm}}$ & 29 & & 16 & & 19 & & 13 & & 10 & \\
\hline & $m_{\tilde{\chi}_{2}^{ \pm}}$ & 221 & & 131 & & 13 & & 18 & & 26 & \\
\hline & & Gen. 1,2 & Gen. 3 & Gen. 1,2 & Gen. 3 & Gen. 1,2 & Gen. 3 & Gen. 1,2 & Gen. 3 & Gen. 1,2 & Gen. 3 \\
\hline$\stackrel{2}{\approx}$ & $m_{\tilde{u}_{1}}$ & 1712 & 1542 & 1040 & 921 & 1013 & 987 & 1283 & 717 & 1677 & 1107 \\
\hline 苟 & $m_{\tilde{u}_{2}}$ & 1704 & 2042 & 1038 & 1164 & 1006 & 1336 & 1289 & 1260 & 1683 & 1860 \\
\hline$\frac{0}{0}$ & $m_{\tilde{d}_{1}}$ & 1714 & 2037 & 1043 & 1150 & 1016 & 1316 & 1285 & 1223 & 1678 & 1838 \\
\hline$\frac{\sqrt{3}}{=}$ & $m_{\tilde{d}_{2}}$ & 1651 & 2321 & 1036 & 1341 & 888 & 1379 & 1351 & 1702 & 1688 & 2364 \\
\hline Е్ & $m_{\tilde{e}_{1}}$ & 1532 & 2192 & 970 & 1227 & 769 & 1182 & 1334 & 1696 & 1694 & 2356 \\
\hline 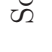 & $m_{\tilde{e}_{2}}$ & 1586 & 2206 & 968 & 1305 & 901 & 1228 & 1256 & 1750 & 1687 & 2370 \\
\hline & $m_{\tilde{\nu}}$ & 1530 & 2196 & 966 & 1296 & 764 & 1202 & 1331 & 1746 & 1692 & 2366 \\
\hline & $\delta \rho$ & $8.5 \times$ & $10^{-6}$ & $3.0 \times$ & $0^{-5}$ & $2.3 \times$ & $0^{-5}$ & $2.1 \times$ & $10^{-5}$ & $7.2 \times$ & $10^{-6}$ \\
\hline & $\delta(g-2)_{\mu}$ & $6.0 \times 1$ & $0^{-11}$ & $3.9 \times 1$ & $0^{-10}$ & $5.5 \times$ & $0^{-10}$ & $7.0 \times$ & $0^{-11}$ & $1.2 \times$ & $0^{-11}$ \\
\hline 0 & $B R(b \rightarrow s \gamma)$ & $3.7 \times$ & $10^{-4}$ & $3.9 \times$ & $0^{-4}$ & $3.9 \times$ & $0^{-4}$ & $3.6 \times$ & $10^{-4}$ & $3.7 \times$ & $10^{-4}$ \\
\hline$\underset{\mathbb{E}}{+}$ & $B R\left(B_{s} \rightarrow \mu^{+} \mu^{-}\right)$ & $3.1 \times$ & $10^{-9}$ & $2.7 \times$ & $0^{-9}$ & $2.9 \times$ & $0^{-9}$ & $3.1 \times$ & $10^{-9}$ & $3.1 \times$ & $10^{-9}$ \\
\hline 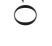 & $m_{L M M}$ & 27 & & 17 & & 13 & & 53 & & 48 & \\
\hline & $m_{n L M M}$ & 416 & & 256 & & 227 & & 272 & & 367 & \\
\hline
\end{tabular}

Table 7: Weak scale observables, with no contribution from gauge mediation: $n_{3}=n_{2}=n_{1}=0$, see Eqn. 78. We have listed the mass eigenstates of the squarks and sleptons. Note that for light generations, $m_{\tilde{u}_{1}} \approx m_{\tilde{u}_{L}}$, etc. The last two rows give the lightest massive modulus $\left(m_{L M M}\right)$ [mostly Kähler modulus $(\operatorname{Re} T)]$ and the next to lightest massive modulus $\left(m_{n L M M}\right)$ [mostly the dilaton $(\operatorname{Re} S)$ ]. All other moduli have mass $\gtrsim 100 \mathrm{TeV}$. 
In all cases the lightest MSSM particle is mostly ( $\gtrsim 99 \%$ ) bino (see Table 8). We note that this is generically true in the models, even when there are contributions from gauge mediation. The gauge mediated contributions in Eqn. (78) do not appreciably change the composition of the LSP, which one can check with the solutions in Table 7 of Reference [97.

We have evaluated other low energy observables using micrOMEGAs [106]. As expected, the bino LSP overcloses the universe, giving $\Omega_{\mathrm{DM}}>>\Omega_{\mathrm{DM}}^{\mathrm{OBS}} \approx$ 0.2. The calculated values for the following observables are given in the last few rows of Table 7. Corrections to the $\rho$ parameter are very small. Corrections to $(g-2)_{\mu}$ are significant in Cases 2 and 3 which is not surprising since these are the two cases with the lightest sleptons for the first two families. We also display the results for $B R(b \rightarrow s \gamma)$ and $B R\left(B s_{s} \rightarrow \mu^{+} \mu^{-}\right)$. The result for $B R(b \rightarrow s \gamma)$ is within the $2 \sigma$ experimental bound (see [86] and references therein). Given the small chargino masses and the large values of $\mu$ and the squark and CP odd Higgs masses, we obtain a branching ratio $B R\left(B_{s} \rightarrow \mu^{+} \mu^{-}\right)$consistent with the standard model.

We are not overly concerned about the fact that binos seem to overclose the universe. In some of the heterotic orbifold models the Higgs $\mu$ term vanishes in the supersymmetric limit. Hence there is a Peccei-Quinn symmetry. Supersymmetry breaking effects are expected to shift the moduli VEVs and generate a non-vanishing $\mu$ term; spontaneously breaking the PQ symmetry and producing the standard invisible axion. In fact, it has been shown that PQ axions may be obtained in heterotic orbifold contructions [85]. In such cases it is possible that the bino decays to an axino + photon leaving an axino dark matter candidate [107, 108, 109].

However another, perhaps more important, cosmological effect must be considered. All 5 cases have a gravitino with mass less than $3 \mathrm{TeV}$. Thus there is most likely a gravitino problem. In addition the lightest moduli mass is of order (Table 7) several $100 \mathrm{~s}$ GeV. Thus there is also a cosmological moduli problem. But there is hope. The next lightest massive modulus [nLMM] has, in all cases, a mass above $20 \mathrm{TeV}$. A detailed cosmological analysis is beyond the scope of this paper. However, it is possible that when cosmological temperatures are of order $m_{\mathrm{nLMM}}$, the universe becomes nLMM dominated. By the time the nLMM decays all matter is diluted and then the universe reheats to temperatures above the scale of big bang nucleosynthesis (for example, see [87]). Thus it is possible that the nLMM solves both the gravitino and light moduli problems. Of course, then the issue of obtaining the correct baryon asymmetry of the universe and the dark 


\begin{tabular}{|cc|c|c|c|c|c|c|c|c|c|c|}
\hline \multirow{2}{*}{ Case } & \multicolumn{2}{|c|}{1} & \multicolumn{2}{|c|}{2} & \multicolumn{2}{|c|}{3} & \multicolumn{2}{|c|}{4} & \multicolumn{2}{c|}{5} \\
\cline { 2 - 12 } & & $\mu<0$ & $\mu>0$ & $\mu<0$ & $\mu>0$ & $\mu<0$ & $\mu>0$ & $\mu<0$ & $\mu>0$ & $\mu<0$ & $\mu>0$ \\
\hline \multirow{2}{*}{$\tan \beta$} & lo & 5 & 6 & 8 & 12 & 9 & 11 & 5 & 4 & 5 & 3 \\
& hi & 38 & 50 & 36 & 50 & 39 & 50 & 32 & 48 & 39 & 50 \\
\hline \multirow{2}{*}{$m_{h^{0}}$} & lo & 113.5 & 113.2 & 112.4 & 112.4 & 112.4 & 112.4 & 112.4 & 116.2 & 113.5 & 112.5 \\
& hi & 117.4 & 117.2 & 113.7 & 113.4 & 113.6 & 113.7 & 120.5 & 121.8 & 120.8 & 121.9 \\
\hline Neut. & & bino & bino & bino & bino & bino & bino & bino & bino & bino & bino \\
comp. & & $\gtrsim 99 \%$ & $\gtrsim 99 \%$ & $\gtrsim 99 \%$ & $\gtrsim 99 \%$ & $\gtrsim 99 \%$ & $\gtrsim 99 \%$ & $\gtrsim 99 \%$ & $\gtrsim 99 \%$ & $\gtrsim 99 \%$ & $\gtrsim 99 \%$ \\
\hline$m_{\tilde{\chi}_{1}^{0}}$ & lo & 149.1 & 148.5 & 82.5 & 82.0 & 98.6 & 98.2 & 68.9 & 67.3 & 53.6 & 51.5 \\
$(\mathrm{GeV})$ & hi & 151.5 & 149.9 & 84.0 & 82.9 & 99.8 & 98.7 & 69.6 & 70.3 & 55.1 & 55.9 \\
\hline$m_{\tilde{\chi}_{1}^{ \pm}}$ & lo & 290.4 & 291.3 & 162.3 & 162.3 & 193.5 & 193.6 & 139.4 & 134.3 & 110.3 & 103.3 \\
$(\mathrm{GeV})$ & hi & 298.8 & 293.7 & 167.1 & 163.7 & 197.7 & 194.5 & 141.0 & 141.6 & 113.8 & 114.6 \\
\hline \hline
\end{tabular}

Table 8: Scan over $\tan \beta$ and $\operatorname{sgn}(\mu)$.

matter abundance must be addressed. Both can in principle be obtained via non-thermal processes at low temperature.

In Table 8 we analyze the dependence of our results on the value of $\tan \beta$ and $\operatorname{sgn}(\mu)$ with all other input parameters fixed. We find that only the value of the light Higgs mass is sensitive to varying $\tan \beta$. Note the lowest value of $\tan \beta$ is obtained by the Higgs mass bound, while the largest value of the light Higgs mass is obtained with the largest value of $\tan \beta$ (for both signs of $\mu$ ). Additionally, at large $\tan \beta$ for $\mu<0$ the Higgs potential becomes unbounded from below. For $\mu>0$ we limited the analysis to $\tan \beta \leq 50$. The light Higgs mass does not go above $122 \mathrm{GeV}$ for $\tan \beta \leq 50$.

\section{Conclusions}

As a candidate theory of all fundamental interactions, string theory should admit at least one example of a four-dimensional vacuum which contains particle physics and early universe cosmology consistent with the two standard models. In this context, the recently found "mini-landscape" of heterotic orbifold constructions [4, 5, 6, 9, 10] provide us with very promising fourdimensional perturbative heterotic string vacua. Their low-energy effective field theory was shown to resemble that of the MSSM, assuming non-zero VEVs for certain blow-up moduli fields which parametrize resolutions of the orbifold fixed points along $F$ - and $D$-flat directions in global supersymmetry. 
In this paper we have dealt with the task of embedding the globally supersymmetric constructions of the heterotic "mini-landscape" into supergravity and then stabilizing the moduli of these compactifications, including their orbifold fixed point blow-up moduli. The blow-up moduli appear as chiral superfields contained in the twisted sectors of the orbifolded heterotic string theory. They are singlets under all standard model gauge groups, but are charged under several unwanted $U(1)$ gauge symmetries, including the universal anomalous $U(1)_{A}$ gauge symmetry of the heterotic string. Note, moduli stabilization of string compactifications is a crucial precondition for comparing to low energy data, as well as for analyzing any early universe cosmology, such as inflation, in a given construction.

Section 2 served the purpose of reviewing the ingredients and structure of the heterotic $4 \mathrm{~d} N=1$ supergravity inherited from orbifold compactifications of the $10 \mathrm{~d}$ perturbative $E_{8} \otimes E_{8}$ heterotic string theory. The general structure of these compactifications results in:

i) a standard no-scale Kähler potential for the bulk volume and complex structure moduli, as well as the dilaton, together with

ii) gaugino condensation in the unbroken sub-group of the hidden $E_{8}$, and

iii) the fact that the non-perturbative (in the world-sheet instanton sense) Yukawa couplings among the twisted sector singlet fields contain terms explicitly breaking the low-energy $U(1)_{R^{-} \text {-symmetry. }}$

We have shown in Section 3 that these three general ingredients, present in all of the "mini-landscape" constructions, effectively realize a KKLT-like setup for moduli stabilization. Here, the existence of terms explicitly break-

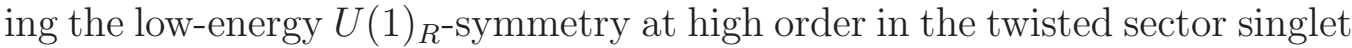
fields is the source of the effective small term $w_{0}$ in the superpotential, which behaves like a constant with respect to the heterotic dilaton [81]. Utilizing this, the presence of just a single condensing gauge group in the hidden sector (in contrast to the racetrack setups in the heterotic literature) suffices to stabilize the bulk volume $T$ (and, by extension, also the bulk complex structure moduli $U$ ), as well as the dilaton $S$ at values $\langle\operatorname{Re} T\rangle \sim 1.1-1.6$ and $\langle\operatorname{Re} S\rangle \sim 2$. These are the values suitable for perturbative gauge coupling unification into $S U(5)$ - and $S O(10)$-type GUTs distributed among the orbifold fixed points. Note, we have shown this explicitly for the case one $T$ modulus and a dilaton, however, we believe that all bulk moduli will be stabilized near their self-dual points [76, 82]. 
At the same time, the near-cancelation of the $D$-term of the universal anomalous $U(1)_{A}$-symmetry stabilizes non-zero VEVs for certain gauge invariant combinations of twisted sector singlet fields charged under the $U(1)_{A}$. This feature in turn drives non-vanishing $F$-terms for some of the twisted sector singlet fields. Thus, together with the $F$-terms of the bulk volume moduli inherited from modular invariance, it is sufficient to uplift the AdS vacuum to near-vanishing cosmological constant.

The structure of the superpotential discussed in this paper, $\mathcal{W} \sim w_{0} e^{-b T}+$ $\phi_{2} e^{-a S-b_{2} T}$, behaves like a 'hybrid KKLT' with a single-condensate for the dilaton $\mathrm{S}$, but as a racetrack for the $T$ and, by extension, also for $U$ moduli. An additional matter $F_{\phi_{2}}$ term driven by the cancelation of the anomalous $U(1)_{A} D$-term seeds successful up-lifting.

We note the fact that the effective constant term in the superpotential, $w_{0}$, does not arise from a flux superpotential akin to the type IIB case. This leaves open (for the time being) the question of how to eventually fine-tune the vacuum energy to the $10^{-120}$-cancelation necessary.

Section 4 then serves to demonstrate how the success of stabilizing the bulk moduli and breaking supersymmetry in the $F$-term sector, driven by the $U(1)_{A} D$-term cancelation, transmits itself to the chiral singlet fields from the untwisted and twisted sectors of the orbifold compactification which contain, among others, the blow-up moduli associated with the orbifold fixed points. The effects from the bulk moduli stabilization and supersymmetry breaking, transmitted through supergravity, generically suffice to stabilize all of the twisted sector singlet fields at non-zero VEVs. This property was assumed in the original "mini-landscape" construction in order to decouple the nonMSSM vector-like exotic matter, and our arguments provide the first step towards a self-consistent justification for these assumptions.

In Section 5 we estimate the structure of the soft terms from the moduli sector supersymmetry breaking at the high scale. We find that the contributions from high-scale gauge mediation are subdominant (although not parametrically suppressed) compared to the gravity mediated contributions. Upon RGE running the high-scale soft terms to the weak scale using softSUSY, we obtain several benchmark patterns of sparticle and Higgs masses (see Table 7). The low-energy spectrum features an allowed window of $\tan \beta$ values for $m_{3 / 2}<5 \mathrm{TeV}$. It generically contains a light chargino/ neutralino spectrum and heavy squarks and sleptons. The lightest MSSM partner, in the 5 benchmark cases studied, is given by a bino $(>99 \%)$ with mass $\gtrsim 52 \mathrm{GeV}$. If this were the LSP, it would yield a dark matter abun- 
dance which over closes the universe, however, the "mini-landscape" models offer some possible resolutions. One possibility is that the bino decays into an axino, the partner of the invisible axion responsible for canceling the $\theta$ angle of QCD, which is present in many of the "mini-landscape" setups [85]. We have also considered an alternative possibility that the late decay of the next to lightest massive modulus might ameliorate or solve the cosmological gravitino and moduli problem. This would then dilute the above mentioned cosmological abundance of binos. Of course, the non-thermal production of dark matter and a baryon asymmetry must then be addressed. Note, however, the resolution of these cosmological questions are beyond the scope of the present paper.

Summarizing, we have given a mechanism for moduli stabilization and supersymmetry breaking for the perturbative heterotic orbifold compactifications. It relies on the same variety and number of effective ingredients as the KKLT construction of type IIB flux vacua and thus represents a significant reduction in necessary complexity, compared to the multi-condensate racetrack setups utilized so far. When applied to a simplified analog of the "mini-landscape" heterotic orbifold compactifications, which give the MSSM at low energies, it leads to fully stabilized $4 \mathrm{~d}$ heterotic vacua with broken supersymmetry and a small positive cosmological constant. Moreover, most of the low energy spectrum could be visible at the LHC.

We leave some important questions like the problem of the full fine-tuning of the vacuum energy to near-vanishing, or the existence of an inflationary cosmology within these stabilized "mini-landscape" constructions for future work. Further study is also warranted with respect to potential cosmological moduli and gravitino problems that may be associated with sub-100 TeV moduli and gravitino mass values (see e.g. [114]). Finally, the numerical evaluation of any particular "mini-landscape" vacuum requires analyzing the supergravity limit with three bulk moduli, $T$, one bulk complex structure modulus, $U$, and of order 50 blow-up moduli. A detailed analysis of this more realistic situation would require a much better handle on the moduli space of heterotic orbifold models than is presently available. 


\section{Acknowledgements}

A.W. would like to thank the Physics Department at the Ohio State University for their warm hospitality, where part of this work was completed. B.D. and S.R. would like to thank the Stanford Institute for Theoretical Physics for their hospitality, where the bulk of this work was completed. Also B.D., S.R. and A.W. thank the KITP at UC Santa Barbara for support. We are also indebted to Patrick Vaudrevange for aid with the superpotential for Model IA. B.D. and S.R. are supported by DOE grant DOE/ER/01545-884. A.W. is supported in part by the Alexander-von-Humboldt foundation, as well as by NSF grant PHY-0244728. This research was supported in part by the National Science Foundation under Grant No. NSF PHY05-51164. We would also like to thank K. Bobkov and N. Craig for useful discussions. S.R. would also like to thank W. Buchmüller, J. Conlon, E. Dudas, G. Dvali, T. Kobayashi, D. Lüst, H.P. Nilles, M. Ratz, S. Ramos-Sanchez, and G.G. Ross for discussions. 


\section{A A Different Racetrack.}

The form of the gaugino condensate, given in Eqn. (35), ensures that the non-perturbative part of the superpotential is invariant under the modular group $\operatorname{SL}(2, \mathbb{Z})$. In deriving the form of $\mathcal{W}_{\mathrm{NP}}$, however, we have neglected the fact that the presence of discrete Wilson lines often break the modular group $\mathrm{SL}(2, \mathbb{Z})$ to one of its subgroups. It has been noted 66] that turning on one or more Wilson lines breaks the modular group $\mathrm{SL}(2, \mathbb{Z})$ down to one of its subgroups. Define the subgroup $\Gamma_{0}(p) \subset \mathrm{SL}(2, \mathbb{Z})$. The subgroup is defined as the set of $2 \times 2$ matrices such that 23

$$
\begin{aligned}
\mathcal{M} & \equiv\left(\begin{array}{ll}
a & b \\
c & d
\end{array}\right), \\
a d-c b & =1, \\
a, b, c, d & \in \mathbb{Z}, \\
c & \equiv 0 \bmod p, p \in \mathbb{P},
\end{aligned}
$$

where $\mathbb{P}$ is the set of prime integers. Under this subgroup, then, the invariant function is a linear combination of Dedekind $\eta$ functions:

$$
f_{p}(\tau)=\frac{1}{p} \sum_{\lambda=0}^{p-1} \eta\left(\frac{\tau+\lambda}{p}\right) .
$$

\section{B The Role of Holomorphic Monomials}

Supersymmetry can be broken by either $F$ terms or $D$ terms. In a generic supersymmetric gauge theory, $D=0$ is satisfied only along special directions in moduli space. These directions are described by holomorphic, gauge invariant monomials (HIMs) [110, 111, 112. The moduli space of a general heterotic string model is significantly more complex than that of our simple models. Not only are there many more fields in the picture, there are also many more gauge groups.

Consider a theory with gauge symmetry $\mathrm{U}(1)^{\rho} \otimes \mathrm{U}(1)_{A}$, where $A$ stands for anomalous. The $D=0$ constraints are

$$
D_{a \neq A} \sim \sum_{i} q_{i}^{a}\left|\phi_{i}\right|^{2}=0 .
$$

\footnotetext{
${ }^{23} \mathrm{~A}$ detailed mathematical treatment of the modular functions can be found in Reference [113.
} 
A generic HIM can be written in terms of fields $\phi_{i}$ with charges $q_{i}^{j}$

$$
\mathcal{H}\left[\phi_{i}\right]=\prod_{i} \phi_{i}^{n_{i}}, n_{i}>0,
$$

such that

$$
\sum_{i} n_{i} q_{i}^{j}=0, \quad \forall j \neq A .
$$

The requirement that $n_{i}>0$ is a reflection of the holomorphicity of $\mathcal{H}$, while the requirement that the sum over $n_{i}$ (weighted by the charges) vanishes is a reflection of the gauge invariance. The general HIM in Eqn. (102) relates the VEVs of the fields $\phi$ as follows:

$$
\frac{\left|\phi_{1}\right|}{\sqrt{n_{1}}}=\frac{\left|\phi_{2}\right|}{\sqrt{n_{2}}}=\cdots .
$$

Given this relationship, one can show that the Eqns. (101) can be satisfied. Notice that no scale is introduced in Eqn. (104): the HIMs (in general) only constrain the relative magnitudes of the $\phi$ VEVs, and gives no information about their phases or their absolute magnitudes.

The procedure for dealing with an anomalous $\mathrm{U}(1)_{A}$ works the same way. Instead of Eqn. (101), one has

$$
D_{A} \sim \sum_{i} q_{i}^{A}\left|\phi_{i}\right|^{2}+\xi=0,
$$

and we will assume that $\xi>0$. In this case, one needs to find a monomial which is holomorphic and gauge invariant under all of the $\rho \mathrm{U}(1)$ factors, but which carries a net negative charge under the anomalous $\mathrm{U}(1)_{A}[110$, 111, 112. The situation is different than the case with non-anomalous symmetries, as a mass scale is introduced into the problem.

In a heterotic string orbifold, the FI term is generated by the mixed gaugegravitational anomaly, and is canceled by the Green-Schwarz mechanism, which forces singlets to get VEVs of order the FI scale (typically $\sim M_{\mathrm{S}}$ ). Usually, several singlets participate in this cancellation, all receiving VEVs of the same order. In the "mini-landscape" models 9], supersymmetric vacua were obtained, prior to the consideration of any non-perturbative effects. A holomorphic gauge invariant monomial was found which is invariant under all other $U(1) \mathrm{s}$ but with net charge under $U(1)_{A}$ opposite to that of the 
FI term. This composite field necessarily gets a non-zero VEV to cancel the FI term. Our field $\phi_{2}$ in the simple model gives mass to the vector-like exotics of the hidden sector and thus it also appears in the non-perturbative superpotential. In a more general heterotic model, $\phi_{2}$ would be replaced by an HIM which also cancels the FI term.

\section{References}

[1] G. B. Cleaver, A. E. Faraggi, and D. V. Nanopoulos, "String derived MSSM and M-theory Unification," Phys. Lett. B455 (1999) 135-146, arXiv:hep-ph/9811427.

[2] V. Braun, Y.-H. He, B. A. Ovrut, and T. Pantev, "A heterotic standard model," Phys. Lett. B618 (2005) 252-258,

arXiv:hep-th/0501070.

[3] M. Cvetic, T. Li, and T. Liu, "Standard-like Models as Type IIB Flux Vacua," Phys. Rev. D71 (2005) 106008, arXiv:hep-th/0501041.

[4] W. Buchmüller, K. Hamaguchi, O. Lebedev, and M. Ratz, "Supersymmetric standard model from the heterotic string," Phys. Rev. Lett. 96 (2006) 121602, arXiv:hep-ph/0511035.

[5] W. Buchmüller, K. Hamaguchi, O. Lebedev, and M. Ratz, "Supersymmetric standard model from the heterotic string. II," Nucl. Phys. B785 (2007) 149-209, arXiv: hep-th/0606187.

[6] O. Lebedev et al., "A mini-landscape of exact MSSM spectra in heterotic orbifolds," Phys. Lett. B645 (2007) 88-94. arXiv:hep-th/0611095.

[7] J. E. Kim, J.-H. Kim, and B. Kyae, "Superstring standard model from $\mathbb{Z}_{12}$-I orbifold compactification with and without exotics, and effective $R$ - parity," JHEP 06 (2007) 034, arXiv: hep-ph/0702278.

[8] C.-M. Chen, T. Li, V. E. Mayes, and D. V. Nanopoulos, "A Realistic World from Intersecting D6-Branes," Phys. Lett. B665 (2008) 267-270, arXiv:hep-th/0703280. 
[9] O. Lebedev et al., "The Heterotic Road to the MSSM with $R$ parity," Phys. Rev. D77 (2008) 046013, arXiv:0708.2691 [hep-th].

[10] O. Lebedev, H. P. Nilles, S. Ramos-Sanchez, M. Ratz, and P. K. S. Vaudrevange, "Heterotic mini-landscape (II): completing the search for MSSM vacua in a $\mathbb{Z}_{6}$-II orbifold," Phys. Lett. B668 (2008) 331-335, arXiv:0807.4384 [hep-th].

[11] C. Beasley, J. J. Heckman, and C. Vafa, "GUTs and Exceptional Branes in F-theory - I," JHEP 01 (2009) 058, arXiv:0802.3391 [hep-th].

[12] R. Donagi and M. Wijnholt, "Model Building with F-Theory," arXiv:0802.2969 [hep-th].

[13] C. Beasley, J. J. Heckman, and C. Vafa, "GUTs and Exceptional Branes in F-theory - II: Experimental Predictions," JHEP 01 (2009) 059, arXiv:0806.0102 [hep-th].

[14] R. Donagi and M. Wijnholt, "Breaking GUT Groups in F-Theory," arXiv:0808.2223 [hep-th].

[15] R. Blumenhagen, V. Braun, T. W. Grimm, and T. Weigand, "GUTs in Type IIB Orientifold Compactifications," Nucl. Phys. B815, 1 (2009), arXiv:0811.2936 [hep-th].

[16] J. L. Bourjaily, "Local Models in F-Theory and M-Theory with Three Generations," arXiv:0901.3785 [hep-th].

[17] H. Hayashi, T. Kawano, R. Tatar and T. Watari, "Codimension-3 Singularities and Yukawa Couplings in F-theory," Nucl. Phys. B823, 47 (2009), arXiv:0901.4941 [hep-th].

[18] J.-H. Huh, J. E. Kim, and B. Kyae, "SU(5) flip $\times S U(5)^{\prime}$ from $\mathbb{Z}_{12}$-I," Phys. Rev. D80, 115012 (2009), arXiv:0904.1108 [hep-ph].

[19] J. Marsano, N. Saulina and S. Schafer-Nameki, "Monodromies, Fluxes, and Compact Three-Generation F-theory GUTs," JHEP 0908, 046 (2009) arXiv:0906.4672 [hep-th]. 
[20] H. P. Nilles, S. Ramos-Sanchez, M. Ratz and P. K. S. Vaudrevange, "From strings to the MSSM," Eur. Phys. J. C59, 249 (2009) arXiv:0806.3905 [hep-th].

[21] S. Raby, "String Model Building," AIP Conf.Proc. 1200:235-245,2010, arXiv:0911.1172 [hep-ph].

[22] S. G. Nibbelink, T. W. Ha and M. Trapletti, "Toric Resolutions of Heterotic Orbifolds," Phys. Rev. D77, 026002 (2008) arXiv:0707.1597 [hep-th].

[23] S. G. Nibbelink, J. Held, F. Ruehle, M. Trapletti and P. K. S. Vaudrevange, "Heterotic $\mathbb{Z}_{6}$-II MSSM Orbifolds in Blowup," JHEP 0903, 005 (2009) arXiv:0901.3059 [hep-th].

[24] O. Lebedev et al., "Low Energy Supersymmetry from the Heterotic Landscape," Phys. Rev. Lett. 98 (2007) 181602, arXiv:hep-th/0611203.

[25] S. B. Giddings, S. Kachru, and J. Polchinski, "Hierarchies from fluxes in string compactifications," Phys. Rev. D66 (2002) 106006, arXiv:hep-th/0105097.

[26] S. Kachru, R. Kallosh, A. Linde, and S. P. Trivedi, "De Sitter vacua in string theory," Phys. Rev. D68 (2003) 046005. arXiv:hep-th/0301240.

[27] J. J. Heckman, J. Marsano, N. Saulina, S. Schafer-Nameki and C. Vafa, "Instantons and SUSY breaking in F-theory," arXiv:0808.1286 [hep-th].

[28] J. Marsano, N. Saulina and S. Schafer-Nameki, "Gauge Mediation in F-Theory GUT Models," Phys. Rev. D80, 046006 (2009) [arXiv:0808.1571 [hep-th]].

[29] J. J. Heckman and C. Vafa, "F-theory, GUTs, and the Weak Scale," JHEP 0909, 079 (2009) arXiv:0809.1098 [hep-th].

[30] P. Binetruy and E. Dudas, "Gaugino condensation and the anomalous U(1)," Phys. Lett. B389, 503 (1996), [arXiv:hep-th/9607172]. 
[31] G. R. Dvali and A. Pomarol, "Anomalous U(1) as a mediator of supersymmetry breaking," Phys. Rev. Lett. 77, 3728 (1996), [arXiv:hep-ph/9607383].

[32] G. R. Dvali and A. Pomarol, "Anomalous U(1), gauge-mediated supersymmetry breaking and Higgs as pseudo-Goldstone bosons," Nucl. Phys. B522, 3 (1998), [arXiv:hep-ph/9708364].

[33] Z. Lalak, "Anomalous D-term, dynamical supersymmetry breaking and dynamical gauge couplings," Nucl. Phys. B521, 37 (1998), [arXiv:hep-ph/9708410].

[34] B. de Carlos, S. Gurrieri, A. Lukas and A. Micu, "Moduli stabilisation in heterotic string compactifications," JHEP 0603, 005 (2006), [arXiv:hep-th/0507173].

[35] K. Choi and K. S. Jeong, "Supersymmetry breaking and moduli stabilization with anomalous U(1) gauge symmetry," JHEP 0608, 007 (2006), [arXiv:hep-th/0605108].

[36] E. Dudas and Y. Mambrini, "Moduli stabilization with positive vacuum energy," JHEP 0610, 044 (2006), [arXiv:hep-th/0607077].

[37] Z. Lalak, O. J. Eyton-Williams and R. Matyszkiewicz, "F-term uplifting via consistent D-terms," JHEP0705, 085 (2007), [arXiv:hep-th/0702026].

[38] E. Dudas, Y. Mambrini, S. Pokorski and A. Romagnoni, "Moduli stabilization with Fayet-Iliopoulos uplift," JHEP 0804, 015 (2008), [arXiv:0711.4934 [hep-th]].

[39] D. Gallego and M. Serone, "Moduli Stabilization in non-Supersymmetric Minkowski Vacua with Anomalous U(1) Symmetry," JHEP 08 (2008) 025, arXiv:0807.0190 [hep-th].

[40] E. Dudas, Y. Mambrini, S. Pokorski, A. Romagnoni and M. Trapletti, "Gauge vs. Gravity mediation in models with anomalous U(1)'s," JHEP 0903, 011 (2009), [arXiv:0809.5064 [hep-th]].

[41] M. B. Green and J. H. Schwarz, "Anomaly Cancellation in Supersymmetric $D=10$ Gauge Theory and Superstring Theory," Phys. Lett. B149 (1984) 117-122. 
[42] P. Fayet and J. Iliopoulos, "Spontaneously Broken Supergauge Symmetries and Goldstone Spinors," Phys. Lett. B51 (1974) 461-464.

[43] M. Dine, N. Seiberg, and E. Witten, "Fayet-Iliopoulos Terms in String Theory," Nucl. Phys. B289 (1987) 589.

[44] J. J. Atick, L. J. Dixon, and A. Sen, "String Calculation of Fayet-Iliopoulos D Terms in Arbitrary Supersymmetric Compactifications," Nucl. Phys. B292 (1987) 109-149.

[45] M. Dine, I. Ichinose, and N. Seiberg, "F Terms and D Terms in String Theory," Nucl. Phys. B293 (1987) 253.

[46] T. Kobayashi, S. Raby, and R.-J. Zhang, "Searching for realistic 4d string models with a Pati-Salam symmetry: Orbifold grand unified theories from heterotic string compactification on a $\mathbb{Z}_{6}$ orbifold," Nucl. Phys. B704 (2005) 3-55, arXiv:hep-ph/0409098.

[47] E. Witten, "Dimensional Reduction of Superstring Models," Phys. Lett. B155 (1985) 151.

[48] S. Ferrara, C. Kounnas and M. Porrati, "General Dimensional Reduction Of Ten-Dimensional Supergravity And Superstring," Phys. Lett. B181, 263 (1986).

[49] M. Cvetic, J. Louis and B. A. Ovrut, "A String Calculation of the Kahler Potentials for Moduli of Z(N) Orbifolds," Phys. Lett. B206, 227 (1988).

[50] A. D. Shapere and F. Wilczek, "Selfdual Models with Theta Terms," Nucl. Phys. B320, 669 (1989).

[51] S. Ferrara, D. Lust, A. D. Shapere and S. Theisen, "Modular Invariance in Supersymmetric Field Theories," Phys. Lett. B225, 363 (1989).

[52] J. Lauer, J. Mas and H. P. Nilles, "Duality and the role of nonperturbative effects on the world sheet," Phys. Lett. B226, 251 (1989).

[53] E. J. Chun, J. Mas, J. Lauer and H. P. Nilles, "Duality and Landau-Ginzburg models," Phys. Lett. B233, 141 (1989). 
[54] S. Ferrara, D. Lust and S. Theisen, "Target Space Modular Invariance and Low-Energy Couplings in Orbifold Compactifications," Phys. Lett. B233, 147 (1989).

[55] J. Lauer, J. Mas and H. P. Nilles, "Twisted Sector Representations Of Discrete Background Symmetries For Two-Dimensional Orbifolds," Nucl. Phys. B351, 353 (1991).

[56] J. Erler, D. Jungnickel and H. P. Nilles, "Space duality and quantized Wilson lines," Phys. Lett. B276, 303 (1992).

[57] J. Erler, D. Jungnickel, J. Lauer and J. Mas, "String emission from twisted sectors: cocycle operators and modular background symmetries," Annals Phys. 217, 318 (1992).

[58] S. Stieberger, D. Jungnickel, J. Lauer and M. Spalinski, "Yukawa Couplings For Bosonic Z(N) Orbifolds: Their Moduli And Twisted Sector Dependence," Mod. Phys. Lett. A7, 3059 (1992) [arXiv:hep-th/9204037].

[59] D. Bailin and A. Love, "Orbifold compactifications of string theory," Phys. Rept. 315, 285 (1999).

[60] L. J. Dixon, V. Kaplunovsky and J. Louis, "On Effective Field Theories Describing $(2,2)$ Vacua of the Heterotic String," Nucl. Phys. B329, 27 (1990).

[61] L.E. Ibañez and D. Lüst, "Duality anomaly cancellation, minimal string unification and the effective low-energy Lagrangian of 4-D strings," Nucl. Phys. B382 (1992) 305-364, arXiv:hep-th/9202046.

[62] S. Hamidi and C. Vafa, "Interactions on Orbifolds," Nucl. Phys. B279 (1987) 465.

[63] J. A. Casas, F. Gomez, and C. Munoz, "Complete structure of Z(n) Yukawa couplings," Int. J. Mod. Phys. A8 (1993) 455-506, arXiv:hep-th/9110060.

[64] J. Erler, D. Jungnickel, M. Spalinski, and S. Stieberger, "Higher twisted sector couplings of Z(N) orbifolds," Nucl. Phys. B397 (1993) 379-416, arXiv:hep-th/9207049. 
[65] P. Vaudrevange, Grand Unification in the Heterotic Braneworld. Ph.D. thesis, Universität Bonn, 2008. http://www.th.physik.uni-bonn.de/nilles/db/thesis/.

[66] A. Love and S. Todd, "Modular symmetries of threshold corrections for Abelian orbifolds with discrete Wilson lines," Nucl. Phys. B481 (1996) 253-288, arXiv:hep-th/9606161.

[67] D. Bailin, A. Love, W. A. Sabra and S. Thomas, "Anisotropic solutions for orbifold moduli from duality invariant gaugino condensates," Mod. Phys. Lett. A9, 2543 (1994) arXiv:hep-th/9405031.

[68] L. J. Dixon, V. Kaplunovsky and J. Louis, "Moduli dependence of string loop corrections to gauge coupling constants," Nucl. Phys. B355, 649 (1991).

[69] L. E. Ibañez, D. Lüst and G. G. Ross, "Gauge Coupling Running In Minimal SU(3) X SU(2) X U(1) Superstring Unification," Phys. Lett. B272, 251 (1991) arXiv:hep-th/9109053.

[70] J. P. Derendinger, S. Ferrara, C. Kounnas and F. Zwirner, "On loop corrections to string effective field theories: Field dependent gauge couplings and sigma model anomalies," Nucl. Phys.B372, 145 (1992).

[71] D. Lüst and C. Muñoz, "Duality invariant gaugino condensation and one loop corrected Kähler potentials in string theory," Phys. Lett. B279 (1992) 272-280, arXiv:hep-th/9201047.

[72] V. Kaplunovsky and J. Louis, "On gauge couplings in string theory," Nucl. Phys. B444 (1995) 191-244, arXiv: hep-th/9502077.

[73] R. Slansky, "Group Theory for Unified Model Building," Phys. Rept. 79 (1981) 1-128.

[74] B. de Carlos, J. A. Casas, and C. Muñoz, "Massive hidden matter and gaugino condensation," Phys. Lett. B263 (1991) 248-254.

[75] I. Affleck, M. Dine, and N. Seiberg, "Dynamical Supersymmetry Breaking in Supersymmetric QCD," Nucl. Phys. B241 (1984) 493-534. 
[76] A. Font, L. E. Ibañez, D. Lüst and F. Quevedo, "Supersymmetry breaking from duality invariant gaugino condensation," Phys. Lett. B245, 401 (1990).

[77] S. Ferrara, N. Magnoli, T. R. Taylor and G. Veneziano, "Duality and supersymmetry breaking in string theory," Phys. Lett. B245, 409 (1990).

[78] H. P. Nilles and M. Olechowski, "Gaugino condensation and duality invariance," Phys. Lett. B248, 268 (1990).

[79] J. A. Casas, Z. Lalak, C. Muñoz, and G. G. Ross, "Hierarchical Supersymmetry Breaking and Dynamical Determination of Compactification Parameters by Non-Perturbative Effects," Nucl. Phys. B347 (1990) 243-269.

[80] B. de Carlos, J. A. Casas, and C. Muñoz, "Supersymmetry breaking and determination of the unification gauge coupling constant in string theories," Nucl. Phys. B399 (1993) 623-653, arXiv: hep-th/9204012.

[81] R. Kappl, et al., "Large hierarchies from approximate $R$ symmetries," Phys. Rev. Lett. 102, 121602 (2009), arXiv:0812.2120 [hep-th].

[82] M. Cvetic, A. Font, L. E. Ibañez, D. Lüst and F. Quevedo, "Target space duality, supersymmetry breaking and the stability of classical string vacua," Nucl. Phys. B361, 194 (1991).

[83] S. Ferrara, C. Kounnas and F. Zwirner, "Mass formulae and natural hierarchy in string effective supergravities," Nucl. Phys. B429, 589 (1994) [Erratum-ibid. B433, 255 (1995)], arXiv:hep-th/9405188.

[84] H. P. Nilles and S. Raby, "Supersymmetry And The Strong CP Problem," Nucl. Phys. B198, 102 (1982).

[85] K. S. Choi, H. P. Nilles, S. Ramos-Sanchez and P. K. S. Vaudrevange, "Accions," Phys. Lett. B675, 381 (2009), [arXiv:0902.3070 [hep-th]].

[86] M. Misiak, "B - ¿ Xs gamma - Current Status," Acta Phys. Polon. B40, 2987 (2009), [arXiv:0911.1651 [hep-ph]]. 
[87] B. S. Acharya, P. Kumar, K. Bobkov, G. Kane, J. Shao and S. Watson, "Non-thermal Dark Matter and the Moduli Problem in String Frameworks," JHEP 0806, 064 (2008), [arXiv:0804.0863 [hep-ph]].

[88] N. V. Krasnikov, "On Supersymmetry Breaking in Superstring Theories," Phys. Lett. B193 (1987) 37-40.

[89] A. Westphal, "de Sitter String Vacua from Kähler Uplifting," JHEP 03 (2007) 102, arXiv:hep-th/0611332.

[90] A. Brignole, L. E. Ibañex, and C. Muñoz, "Towards a theory of soft terms for the supersymmetric Standard Model," Nucl. Phys. B422 (1994) 125-171, arXiv: hep-ph/9308271.

[91] A. Brignole, L. E. Ibañez, C. Muñoz, and C. Scheich, "Some issues in soft SUSY breaking terms from dilaton / moduli sectors," Z. Phys. C74 (1997) 157-170, arXiv:hep-ph/9508258.

[92] Y. Kawamura and T. Kobayashi, "Soft Scalar Masses in String Models with Anomalous U(1) symmetry," Phys. Lett. B375, 141 (1996) [Erratum-ibid. B388, 867 (1996)], [arXiv:hep-ph/9601365].

[93] Y. Kawamura and T. Kobayashi, "Generic formula of soft scalar masses in string models," Phys. Rev. D56, 3844 (1997), [arXiv:hep-ph/9608233].

[94] A. Brignole, L. E. Ibañex, and C. Muñoz, "Soft supersymmetry-breaking terms from supergravity and superstring models," Published In Kane, G.L. (ed.): Perspectives on supersymmetry, 125-148, arXiv:hep-ph/9707209.

[95] P. Binetruy, M. K. Gaillard and B. D. Nelson, "One loop soft supersymmetry breaking terms in superstring effective theories," Nucl. Phys. B604, 32 (2001) arXiv:hep-ph/0011081.

[96] P. Binetruy, A. Birkedal-Hansen, Y. Mambrini and B. D. Nelson, "Phenomenological aspects of heterotic orbifold models at one loop," Eur. Phys. J. C47, 481 (2006) arXiv:hep-ph/0308047.

[97] B. Dundee, S. Raby and A. Wingerter, "Reconciling Grand Unification with Strings by Anisotropic Compactifications," Phys. Rev. D78, (2008) arXiv:0805.4186 [hep-th]. 
[98] G. F. Giudice and A. Masiero, "A Natural Solution to the mu Problem in Supergravity Theories," Phys. Lett. B206, 480 (1988).

[99] J. E. Kim and H. P. Nilles, "The Mu Problem And The Strong CP Problem," Phys. Lett. B138, 150 (1984).

[100] J. E. Kim and H. P. Nilles, "Symmetry principles toward solutions of the mu problem," Mod. Phys. Lett. A9, 3575 (1994) arXiv:hep-ph/9406296.

[101] P. Ko, T. Kobayashi, J. h. Park and S. Raby, "String-derived $D_{4}$ flavor symmetry and phenomenological implications," Phys.Rev. D76, 035005 (2007) [Erratum-ibid. D76, 059901 (2007)] arXiv:0704.2807 [hep-ph] .

[102] Y. Kawamura, "On Low-Energy Theory from General Supergravity," Prog. Theor. Phys. Suppl. 123, 421 (1996), [arXiv:hep-ph/9511334].

[103] B. C. Allanach, "softSUSY: A C++ program for calculating supersymmetric spectra," Comput. Phys. Commun. 143 (2002) 305 arXiv:hep-ph/0104145.

[104] Tevatron Electroweak Working Group, CDF Collaboration, and D0 Collaboration. arXiv:0903.2503 [hep-ex] .

[105] C. Amsler et al. [Particle Data Group], "Review of particle physics," Phys. Lett. B667, 1 (2008).

[106] G. Belanger, F. Boudjema, A. Pukhov and A. Semenov, "Dark matter direct detection rate in a generic model with micrOMEGAs2.1," Comput. Phys. Commun. 180, 747 (2009) arXiv:0803.2360 [hep-ph].

[107] K. Rajagopal, M. S. Turner and F. Wilczek, "Cosmological implications of axinos," Nucl. Phys. B358, 447 (1991).

[108] L. Covi, J. E. Kim and L. Roszkowski, "Axinos as cold dark matter," Phys. Rev. Lett. 82, 4180 (1999) arXiv:hep-ph/9905212.

[109] L. Covi, H. B. Kim, J. E. Kim and L. Roszkowski, "Axinos as dark matter," JHEP 0105, 033 (2001) arXiv: hep-ph/0101009. 
[110] M. A. Luty and W. Taylor, "Varieties of vacua in classical supersymmetric gauge theories," Phys. Rev. D53 (1996) 3399-3405, arXiv:hep-th/9506098.

[111] G. Cleaver, M. Cvetic, J. R. Espinosa, L. L. Everett, and P. Langacker, "Classification of flat directions in perturbative heterotic superstring vacua with anomalous U(1)," Nucl. Phys. B525 (1998) 3-26, arXiv:hep-th/9711178.

[112] B. Dundee, S. Raby, and A. Wingerter, "Addendum to Reconciling Grand Unification with Strings by Anisotropic Compactifications," Phys. Rev. D79 (2009) 047901, arXiv:0811.4026 [hep-th].

[113] T. M. Apostol, Modular functions and Dirichlet series in number theory. Springer-Verlag, New York, 1976.

[114] M. Endo, K. Hamaguchi and F. Takahashi, "Moduli-induced gravitino problem," Phys. Rev. Lett. 96, 211301 (2006) [arXiv:hep-ph/0602061]; S. Nakamura and M. Yamaguchi, "Gravitino production from heavy moduli decay and cosmological moduli problem revived," Phys. Lett. B638, 389 (2006) [arXiv:hep-ph/0602081] 